TRANSACTIONS OF THE

AMERICAN MATHEMATICAL SOCIETY

Volume 239, May 1978

\title{
LIMITING DISTRIBUTIONS FOR BRANCHING RANDOM FIELDS ${ }^{1}$
}

BY

JOSEPH FLEISCHMAN

\begin{abstract}
In this paper we derive limiting distributions for branching Brownian motion. The cases considered are where the state space is (1) the line and (2) the plane where (a) initially there's but one particle and (b) where there's initially a random number of independent particles. In all cases, the branching process is critical and we obtain results for the growth of selectively neutral mutant types. We use moment generating functions to derive these results.
\end{abstract}

1. A. Introduction. In this paper we will discuss a problem whose solution has significance both for its purely mathematical content as well as for its application in population genetics. Thus we will first state the biological problem and then we will formulate the mathematical approach.

One of the major problems of contemporary evolution is to explain the greater than expected genetic diversity found in populations. The classical theory attributes evolution to selective advantage, i.e., the cause of evolution is the spread of mutant types which are selectively advantageous. A more recent theory ascribes evolution to a large number of selectively neutral genes which occur by mutation. Thus, although each new mutant type tends to die out there are enough mutant types around to account for the relatively large number of types present at any given time.

In nature, one generally observes a kind of clustering, i.e., individuals of the same type generally appear close to one another. The classical theory could explain this behavior as being due to geographically varying selection, examples of which are known as clines. The neutralist theory, however, accounts for this situation by the fact that individuals which are located near each other, in general, are more likely to be related. Thus, it is important for us to see if such clustering is actually predicted by a mathematical model which describes the growth of selectively neutral mutant types.

Received by the editors December 1, 1976.

AMS (MOS) subject classifications (1970). Primary 60J80, 60J65; Secondary 92A10, 92A15.

Key words and phrases. Branching process, branching diffusion process, random field, mutation.

'The results of this paper comprise a portion of the author's doctoral dissertation at Yeshiva University. The author expresses his gratitude to Professor Stan Sawyer for guidance and encouragement. 
Suppose we have a large "normal" population in some domain $D$ and a mutation is introduced at a point $a$ in $D$. One can then use a branching process to approximate the growth of the mutant population (cf. Ewens [4, Chapter 7] or Crow and Kimura [3, p. $419+$ ]). A branching process is a Markov process in which all offspring reproduce independently. Thus, in the case of a "rare" mutant type branching processes are a good approximation. This is due to the fact that the presence of a large background of "normal" type individuals makes the likelihood of two mutant types mating sufficiently small. In general, branching processes are useful since one can obtain workable formulas for the various quantities of interest such as means, variances, etc.

In order to obtain geographical information about the growth of populations one can consider branching diffusion processes. In this model, offspring are produced according to a branching process and then proceed to move, branch, move, etc., independently of one another with time being continuous. One can then attempt to find the distribution of the number of offspring of an individual in a given region and then see if there are indications of clustering. One can do this by comparing the rate of decay of a mutant type in the population for a given region, to its rate of decay in the whole state space. We shall consider two cases: the first where initially one mutant individual is injected into the population and the second where a random number of the mutant type, which is uniformly distributed in the plane, is introduced.

B. Mathematical background. Mathematically, the result of this paper is that we determine the limiting population distribution for a branching diffusion process. If one classifies types according to position then this corresponds to the problem of finding the limiting distribution for branching processes with an infinite number of types. By considering time as being discrete (let $t=n \delta$ ) we are, thus, discussing a generalization of the original Galton-Watson problem. Hence this is a continuous time generalization of a multi-type Galton-Watson process.

To formulate the problem mathematically and to outline some background results, let $p_{i}, 0 \leqslant i \leqslant \infty$, correspond to the probability of a single particle producing $i$ offspring at a given branch and let $f(s)$ be its probability generating function, i.e., $f(s)=\Sigma_{n \geqslant 0} p_{n} s^{n}$. If we denote by $f^{(m)}(s)$ the offspring distribution function corresponding to $m$ initial particles each of which branched once, then one can write the branching property as $f^{(m)}(s)=$ $(f(s))^{m}$. The case of selectively neutral mutants corresponds to a critical branching process, i.e., $1=f^{\prime}(1)=\Sigma_{n \geq 0} n p_{n}$-the mean number of offspring.

For critical branching processes (cf. Athreya-Ney [1]) with one particle type and a finite second moment the population tends to die out, specifically, 
$P\left(z_{t}>0\right) \sim 2 / V t f^{\prime \prime}(1)$ and, in fact, one obtains an exponential limit law, i.e.,

$$
\lim _{t \rightarrow \infty} P\left(\frac{2 z_{t}}{V t f^{\prime \prime}(1)}>\lambda \mid z_{t} \neq 0\right)=e^{-\lambda}, \quad \lambda \geqslant 0 .
$$

However, $E\left(z_{t} \mid z_{t} \neq 0\right) \sim c_{1} t\left(c_{1}>0\right)$ which shows that, although the population tends to die out, given that some particle is still alive, there probably are many particles around.

More generally, it was shown by Mullikan [12] that for an arbitrary number of types, given a finite third moment one obtains a similar rate of decay and again one obtains an exponential limit law. However, in order to derive his results he assumes that the expectation operator $M$ satisfies the following two conditions:

A: There exists $n_{0}$ such that $M^{n_{0}}$ is strictly positive.

B: There exists $n_{1}$ such that $M^{n_{1}}$ is a compact operator.

It was shown (cf. Savin and Chistyakov [15], Ogura [13]) that if condition A is not satisfied one does not necessarily obtain an exponential limit law. As a by-product of our results it will be seen that if condition B does not hold one may actually get a different rate of decay.

Branching diffusion processes were first considered by Moyal [11] and Skorokhod [14]. They are multi-type continuous time processes where the movement of the particles in some domain is governed by some Markovian transition density function and which then proceed to move, branch, etc., obeying the branching property and branching at times governed by an additive functional. Equivalently, one can look at it as follows: Suppose at time $t$ there are $n$ particles around, then the $n$-tuple corresponding to the position of the particles is the state of the process at time $t$ where the state space is now $\cup_{n=0}^{\infty} D^{n}=S$ where $D^{n}$ is $n$-fold product of the original state space $D$. It was shown (Ikeda, Nagasawa, and Watanabe [8]) that if the nonbranching part of the process is strong Markov then the branching diffusion process will still be strong Markov. Thus the 2 basic properties of a branching diffusion process are (1) the Markov property and (2) the branching property which can now be written

$$
\bar{T}_{t}\left(\prod_{i=1}^{\infty} f\left(z_{t}^{(i)}\right) \mid z_{0}=\left(a_{1}, \ldots, a_{n}\right)\right)=\prod_{j=1}^{n} \bar{T}_{t}\left(\prod_{i=1}^{\infty} f\left(z_{t}^{(i)}\right) \mid z_{0}=a_{j}\right)
$$

(Ikeda, Nagasawa, and Watanabe [8, p. 255], also Sawyer [16, p. 24]) where $\bar{T}_{t}$ is the expectation operator of the branching diffusion process and $\left(z_{t}^{(1)}, z_{t}^{(2)}, \ldots\right)$ is the present state of the process.

In general, the Markov property implies that the expectation operator $T_{t} f(x)=\int p(t, x, d y) f(y)$ (where the integration is over the state space) forms a semigroup, i.e., $T_{t+s}=T_{t} \circ T_{s}$. The semigroup can be constructed from its 
infinitesimal generator $A f(x)$ where

$$
A f(x)=\lim _{t \rightarrow 0} \frac{T_{t} f(x)-f(x)}{t} .
$$

The construction is accomplished by means of the Hille-Yosida Theorem which states that if $R_{\lambda} f=\int_{0}^{\infty} e^{-\lambda t} T_{\downarrow} f d t$ then under certain general conditions $R_{\lambda} f$ is the unique solution of $(\lambda I-A) R_{\lambda} f=f, R_{\lambda}(\lambda I-A) f=f$.

In the case of a branching diffusion process there are 2 equations relating the expectation operator $\bar{T}_{t} f(x)$ of the branching diffusion process to the expectation operator $T_{t} f(x)$ of the unbranched process. These are the " $S$ equation" (cf. Skorokhod [14]) a nonlinear equation on $D$, and the " $M$ equation" (cf. Moyal [11])

$$
\bar{T}_{\downarrow} f(x)=T_{\downarrow} f(x)+\int_{0}^{t} \int_{s} P\left(x_{\tau} \in d y, \tau \in d r\right) \bar{T}_{t-r} f(y)
$$

where $\tau$ is the first branching time and $x, y \in S \cup\{\triangleleft\}=\hat{S}$, the one point compactification of $S$ and where $f$ is a bounded measurable function on $S$. Thus the $M$-equation is an expression of the strong Markov property applied to the first branching time.

One can also define a mapping $\hat{f}(x), \hat{f}(x)=f\left(x_{1}\right)+f\left(x_{2}\right)+\cdots+f\left(x_{n}\right)$ where $x=\left(x_{1}, \ldots, x_{n}\right)$. It turns out (Ikeda, Nagasawa, and Watanabe [8, Theorem 4.12]) that $C_{t} f(x)=\bar{T} \hat{f}(x)=E_{x}\left[\hat{f}\left(z_{t}\right)\right]$ also forms a semigroup for $f \in B_{0}(D)=\left\{f \mid f\right.$ is bounded, measurable and $\lim _{t \rightarrow 0} C_{t} f(x)=f(x)$, all $\left.x\right\}$, and if $0 \leqslant V(a) \leqslant C$ and provided that $\bar{T}_{t}$ has a density one obtains (Ikeda, Nagasawa, and Watanabe [8, Theorem 4.14], Sawyer [17, Theorem 2.1]) a density $m(t, a, b)$, i.e., $C_{\ell} f(a)=\int m(t, a, b) f(b) d b$, satisfying $\partial m(t, a, b) / \partial t$ $=M_{a} m(t, a, b)$ where $M_{a}=A_{a}+V(a)(K-I)$ where $A_{a}$ is the infinitesimal generator of $T$, beginning at the point $a, V(a) d t$ the probability of a branch in the interval $(t, t+d t)$ and

$$
K f(a)=\sum_{n=0}^{\infty} \int_{D^{n}} \sum_{i=1}^{n} f\left(y_{i}\right) \pi_{n}(a, d y)
$$

where $\pi_{n}(a, d y)$ represents the probability that a single particle starting at $a$ died and was replaced by $n$ particles located at $y=\left(y_{1}, \ldots, y_{n}\right)$. In the case we shall consider, $V(a)=V$ a constant, $\pi_{n}(a, d y)=p_{n} \chi_{(a, \ldots, a)} d y$. In this case $m(t, a, b)=e^{\bar{m} t} p(t, a, b)$ where $p(t, a, b)$ is the transition density function of the unbranched Markov process and $\bar{m}=V\left(\sum_{n=0}^{\infty} n p_{n}-1\right)$. Thus in the critical case $\bar{m}=0$ and $m(t, a, b)=p(t, a, b)$ which in the case of Brownian motion $=e^{-(a-b)^{2} / 2 t} /(2 \pi t)^{d / 2}$ (where $d$ is the dimension of the space). We will denote by $r(A), \frac{1}{2}$ the length of the largest diameter of $A$ and we set

$$
\tilde{n}=V \sum_{n=2}^{\infty} p_{n} n(n-1), \quad \tilde{n}_{r}=V \sum_{n=r}^{\infty} n(n-1) \cdots(n-r+1) p_{n} .
$$


We assume throughout, the growth rate $\tilde{n}_{r}<c^{r}$ (for some $c$, thus implying $\left.p_{n}=O\left(c^{n} / n !\right)\right)$.

Thus, denoting by $N_{A}(t)$ the random variable which corresponds to the number of particles in the bounded set $A$ at time $t$, we have $E_{a}\left(N_{A}(t)\right)=$ $C_{l} \chi_{A}(b)=\int_{A} m(t, a, b) d b$ where $E_{a}()=E\left(\mid x_{0}=a\right)$. In order to obtain our results we need a convenient representation for all the moments of $N_{A}(t)$. Thus we state the following lemma whose proof we defer to Appendix 1.

LEMMA 1.1.

$$
\begin{aligned}
& E_{a}\left[\left(N_{A}(t)\right)^{k}\right]= \int_{A} m(t, a, b) d b \\
&+\sum_{r=2}^{k} \frac{\tilde{n}_{r}}{r !} \sum_{\substack {\left(h_{1}, h_{r}\right) \\
\begin{subarray}{c}{j=1 \\
1<h_{j}<k{ ( h _ { 1 } , h _ { r } ) \\
\begin{subarray} { c } { j = 1 \\
1 < h _ { j } < k } }\end{subarray}} \frac{k !}{h_{1} ! \cdots h_{r} !} \int_{0}^{t} \int_{D} m(t-s, a, b) \\
& \quad E_{b}\left[\left(N_{A}(s)\right)^{h_{1}}\right] \cdots E_{b}\left[\left(N_{A}(s)\right)^{h_{r}}\right] d b d s .
\end{aligned}
$$

Our main results are the limiting distributions for $N_{A}(t)$ in case the particles move about like Brownian motion which is an expected form of movement for biological processes in an infinite homogeneous range (being the limiting case of a random walk). In $\$ 2$ we discuss the case of a single initial particle where the state space $D$ is the plane and our main result is Theorem 2.11:

$$
\lim _{t \rightarrow \infty} \frac{t \ln t \cdot \tilde{n}}{4} F_{t}^{c}(\lambda)=e^{-\lambda}, \quad \lambda>0,
$$

where $F_{t}^{c}(\lambda)=P\left(8 \pi N_{A}(t) / m(A) \tilde{n} \ln t>\lambda\right), m(A)=$ Lebesgue measure of the set $A$, and $\tilde{n}$ is as defined above.

In $\$ 3$ we consider the case of a single particle with $D=(-\infty, \infty)$ and show (Theorem 3.11):

$$
\lim _{t \rightarrow \infty} t \bar{F}_{t}^{c}(\lambda)=F(\lambda) \text { on }\{\lambda: \lambda>0 \text { and } F \text { continuous at } \lambda\}
$$

where $\bar{F}_{t}^{c}(\lambda)=P\left(N_{A}(t) / m(A) \sqrt{t}>\lambda\right)$ and $F(\lambda)$ is a decreasing function on $(0, \infty)$.

In $\$ 4$ we discuss the case of an initial random field of particles with uniform mean density and show (Theorem 4.1), for $D$ the plane

$$
\lim _{t \rightarrow \infty} \frac{\tilde{n} \ln t}{8 \pi} F_{t}^{c}(\lambda)=e^{-\lambda}, \quad \lambda>0,
$$

where 


$$
F_{t}^{c}(\lambda)=P\left(\frac{N_{A}(t) \cdot 8 \pi}{\tilde{n} m(a) \ln t}>\lambda\right)
$$

For $D$ the line we show (Theorem 4.2)

$$
\lim _{t \rightarrow \infty} \frac{\bar{c} \sqrt{t} \tilde{n}}{\sqrt{2 \pi}} \bar{F}_{t}^{c}(\lambda)=\tilde{F}(\lambda), \quad \lambda>0 \text { and } \tilde{F}(x) \text { continuous at } \lambda,
$$

where $\bar{F}_{t}^{c}(\lambda)=P\left(N_{A}(t) \sqrt{2 \pi} / m(A) \tilde{n} \sqrt{t}>\lambda\right), \tilde{F}(\lambda)$ some distribution and $\bar{c}$ a constant.

In the more general case where movement is governed by a stable law, due to the form of the stable densities our method will not work and thus this case remains open. Watanabe [18] considered a similar type of problem in the case of a supercritical process but where $D$ is a bounded domain with an absorbing boundary and thus obtained quite different results.

Our results for a single initial particle with $D$ being the plane indicate a type of clustering effect since for the whole plane earlier results show an asymptotic rate of decay like $1 / t$ whereas our results give an asymptotic rate of decay $O(1 / t \ln t)$ for a bounded region in the plane. Thus it seems to be the case that particles have a tendency to cluster outside any bounded domain. A similar effect turned up in the work of Felsenstein [5] and Sawyer [17].

2. Brownian motion in the plane.

Definition 2.1. Define by induction $\bar{E}_{a}\left(N_{A}(t)\right)=E_{a}\left(N_{A}(t)\right)$, and for $k>1$

$$
\begin{aligned}
& \bar{E}_{a}\left[\left(N_{A}(t)\right)^{k}\right] \\
& \quad=\frac{\tilde{n}}{2} \sum_{j=1}^{k-1}\left(\begin{array}{l}
k \\
j
\end{array}\right) \int_{0}^{t} \int_{D} p(t-s, a, b) \bar{E}_{b}\left[\left(N_{A}(s)\right)^{j}\right] \bar{E}_{b}\left[\left(N_{A}(s)\right)^{k-j}\right] d b d s .
\end{aligned}
$$

For the remainder of the chapter we shall assume $D=$ the plane.

It turns out (Lemma 2.9) that the major contribution to $E_{a}\left(N_{A}(t)\right)^{n}$ comes from $\bar{E}_{a}\left[\left(N_{A}(t)\right)^{n}\right]$ and thus we shall proceed to first derive bounds for $\bar{E}_{a}\left(N_{A}(t)\right)^{n}$. Thus

LEMMA 2.2. $\forall k>0$,

$$
\begin{aligned}
\bar{E}_{a}\left[\left(N_{A}(t)\right)^{k}\right] \geqslant & \frac{k !(m(A))^{k}(\tilde{n})^{k-1}(\ln t)^{k-1}}{(2 \pi)^{k} 4^{k-1} t} \chi_{(t>1)} \\
& \cdot \exp \left[-\frac{\left(a-c_{a}^{\prime}\right)^{2}}{2 t}\right]\left(1-\frac{\Omega_{l}(A, k)}{\ln t}-\frac{a^{2} c(k)}{t \ln t}\right)^{+}
\end{aligned}
$$


where $\left(a-c_{a}^{\prime}\right)^{2}=\max _{c \in A}(a-c)^{2}, \Omega_{l}(A, k)=\left(64\left[(r(A))^{4} \cup 1\right]\right)^{k-1}$, and $c(k)$ $=6^{k-1}$.

Proof. By induction; for $k=1$, by definition $\bar{E}_{a}\left(N_{A}(t)\right)=\int_{A} p(t, a, b) d b$ which in the case of Brownian motion equals

$$
\frac{1}{2 \pi t} \int_{A} \exp \left[-\frac{(a-b)^{2}}{2 t}\right] d b \geqslant \frac{m(A)}{2 \pi t} \chi_{(t>1)} \exp \left[-\frac{\left(a-c_{a}^{\prime}\right)^{2}}{2 t}\right] .
$$

For $k=2$,

$$
\begin{aligned}
\bar{E}_{a}\left[\left(N_{A}(t)\right)^{2}\right]= & \tilde{n} \int_{0}^{t} \int_{D} p(t-s, a, b) \bar{E}_{b}\left(N_{A}(s)\right) \bar{E}_{b}\left(N_{A}(s)\right) d b d s \\
\geqslant & \frac{(m(A))^{2} \tilde{n}}{(2 \pi)^{2}} \int_{1}^{t} \frac{1}{2 \pi(t-s)} \\
& \cdot \frac{1}{s^{2}}\left[\int_{D} \exp \left[-\frac{(a-b)^{2}}{2(t-s)}\right] \cdot \exp \left[-\frac{2\left(b-c_{b}^{\prime}\right)^{2}}{2 s}\right] d b\right] d s
\end{aligned}
$$

by the previous result. Completing a square we get

$$
\begin{aligned}
\int_{D} \exp \left[-\frac{(a-b)^{2}}{2(t-s)}\right] \cdot \exp \left[-\frac{2\left(b-c_{b}\right)^{2}}{2 s}\right] d b \\
>\exp \left[-\frac{\left(a-c_{a}^{\prime}\right)^{2}}{2 t-s}\right] \int_{D} \exp \left[-\left(b-\frac{2 \hat{c}_{b}(t-s)}{2 t-s}\right)^{2}\left(\frac{2 t-s}{2 s(t-s)}\right)\right] d b
\end{aligned}
$$

(for some $\hat{c}_{b} \in A$ ). Changing to polar co-ordinates we obtain

$$
\begin{aligned}
\int_{D} \exp \left[-\left(b-\frac{2 \hat{c}_{b}(t-s)}{2 t-s}\right)^{2}\left(\frac{2 t-s}{2 s(t-s)}\right)\right] d b \\
\geqslant \int_{0}^{2 \pi} \int_{0}^{\infty} \exp \left[-r^{2}\left(\frac{2 t-s}{2 s(t-s)}\right)\right] r d r d \theta \\
-\int_{0}^{2 \pi} \int_{0}^{\infty} \frac{2 r(A)(t-s)}{2 t-s} \exp \left[-\frac{r^{2}}{2}\left(\frac{2 t-s}{s(t-s)}\right)\right] d r d \theta \\
=\left[\frac{2 \pi s(t-s)}{2 t-s}-\frac{4 \pi r(A)(t-s)}{2 t-s} \sqrt{\frac{\pi s(t-s)}{2 t-s}}\right]^{+}
\end{aligned}
$$

Using the estimate,

$$
\exp \left[-\frac{\left(a-c_{a}^{\prime}\right)^{2}}{2 t-s}\right] \geqslant \exp \left[-\frac{\left(a-c_{a}^{\prime}\right)^{2}}{2 t}\right] \cdot\left(1-\frac{\left(a-c_{a}^{\prime}\right)^{2} s}{2 t(2 t-s)}\right)^{+}
$$


we thus obtain,

$$
\begin{gathered}
\bar{E}_{a}\left[\left(N_{A}(t)\right)^{2}\right] \geqslant \frac{(m(A))^{2} \tilde{n}}{(2 \pi)^{2}} \exp \left[-\frac{\left(a-c_{a}^{\prime}\right)^{2}}{2 t}\right] \\
\cdot \int_{1}^{t} \frac{1}{2 \pi(t-s) s^{2}}\left[\frac{2 \pi s(t-s)}{2 t-s}-\frac{4 \pi r(A)(t-s)^{3 / 2}(\pi s)^{1 / 2}}{(2 t-s)^{3 / 2}}\right]^{+} \\
\cdot\left[1-\frac{\left(a-c_{a}^{\prime}\right)^{2} s}{2 t(2 t-s)}\right]^{+} d s .
\end{gathered}
$$

We can bound the integral from below by

$$
\begin{gathered}
{\left[\int_{1}^{t} \frac{1}{s(2 t-s)} d s-\int_{1}^{t} \frac{2 r(A)}{s^{2}(2 t-s)} \sqrt{\frac{\pi s(t-s)}{2 t-s}} d s-\int_{1}^{t} \frac{\left(a-c_{a}^{\prime}\right)^{2}}{2 t(2 t-s)^{2}} d s\right]^{+}} \\
=I_{1}+I_{2}+I_{3} .
\end{gathered}
$$

Clearly, $I_{1}>\ln t / 2 t, I_{2}>-4 r(A) \sqrt{\pi} / t$, and $I_{3} \geqslant-\left(a^{2}+(r(A))^{2}\right) / t^{2}$. Thus,

$$
\begin{aligned}
\bar{E}_{a}\left[\left(N_{A}(t)\right)^{2}\right] & >\exp \left[-\frac{\left(a-c_{a}^{\prime}\right)^{2}}{2 t}\right] \\
\cdot & \frac{(m(A))^{2} \tilde{n} \cdot 2 !}{4(2 \pi)^{2}} \frac{\ln t}{t}\left(1-\frac{\left[2(r(A))^{2}+8 \sqrt{\pi} r(A)\right]}{\ln t}-\frac{2 a^{2}}{t \ln t}\right) X(t>1)
\end{aligned}
$$

verifying the lemma for $k=2$.

Now, assume the lemma correct for $k<n$, then

$$
\begin{aligned}
& \bar{E}_{a}\left[\left(N_{A}(t)\right)^{n}\right]>\frac{(m(A))^{n}(\tilde{n})^{n-1}}{(2 \pi)^{n} 4^{n-2} \cdot 2} \sum_{k=1}^{n-1}\left(\begin{array}{l}
n \\
k
\end{array}\right) k !(n-k) ! \\
& \cdot \int_{1}^{t} \frac{(\ln s)^{n-2}}{2 \pi s^{2}(t-s)}\left[\int_{D} \exp \left[-\frac{(a-b)^{2}}{2(t-s)}\right] \cdot \exp \left[-\frac{2\left(b-c_{b}^{\prime}\right)^{2}}{2 s}\right]\right. \\
& \cdot\left[1-\frac{\Omega_{l}(A, k)}{\ln s}-\frac{b^{2} c(k)}{s \ln s}\right]^{+} \\
&\left.\cdot\left[1-\frac{\Omega_{l}(A, n-k)}{\ln s}-\frac{b^{2} c(n-k)}{\ln s}\right]^{+} d b\right] d s .
\end{aligned}
$$


Since by completing a square we obtain

$$
\begin{aligned}
\int_{D}\left(1-b^{2}\right)^{+} & \exp \left[-\frac{(a-b)^{2}}{2 t-s}\right] \cdot \exp \left[-\frac{2\left(b-c_{b}^{\prime}\right)^{2}}{2 s}\right] d b \\
> & \exp \left[-\frac{\left(a-c_{a}^{\prime}\right)^{2}}{2 t-s}\right] \\
& \cdot \int_{D}\left(1-b^{2}\right)^{+} \exp \left[-\left(b-\frac{a s+2 c_{b}^{\prime}(t-s)}{2 t-s}\right)^{2}\left(\frac{2 t-s}{2 s(t-s)}\right)\right] d b,
\end{aligned}
$$

we must bound the integral

$$
M_{1}=\int_{D} \exp \left[-\left(b-\frac{a s+2 c_{b}^{\prime}(t-s)}{2 t-s}\right)^{2}\left(\frac{2 t-s}{2 s(t-s)}\right)\right] d b
$$

from below and

$$
M_{2}=\int_{D} b^{2} \exp \left[-\left(b-\frac{a s+2 c_{b}^{\prime}(t-s)}{2 t-s}\right)^{2}\left(\frac{2 t-s}{2 s(t-s)}\right)\right] d b
$$

from above. As we showed previously

$$
M_{1} \geqslant\left[\frac{2 \pi s(t-s)}{2 t-s}-\frac{4 \pi r(A)(t-s)}{2 t-s} \sqrt{\frac{\pi s(t-s)}{2 t-s}}\right]^{+} .
$$

By a shift we obtain

$$
\begin{aligned}
M_{2}< & 2 \int_{D} b^{2} \exp \left[-\left(b-\frac{2 \hat{c}_{b}(t-s)}{2 t-s}\right)^{2}\left(\frac{2 t-s}{2 s(t-s)}\right)\right] d b \\
& +2 \int \frac{(a s)^{2}}{(2 t-s)^{2}} \exp \left[-\left(b-\frac{2 \hat{c}_{b}(t-s)}{2 t-s}\right)\left(\frac{2 t-s}{2 s(t-s)}\right)\right] d b \\
& =M_{21}+M_{22} .
\end{aligned}
$$

Since $\hat{c}_{b} \in A$ changing to polar co-ordinates we obtain

$$
\begin{aligned}
M_{21}< & 2 \int_{0}^{2 \pi} \int_{0}^{2 r(A)(t-s) /(2 t-s)} r^{3} d r d \theta+2 \int_{0}^{2 \pi} \int_{2 r(A)(t-s) /(2 t-s)}^{\infty} r^{3} \\
& \cdot \exp \left[-\left(r-\frac{2 r(A)(t-s)}{2 t-s}\right)^{2}\left(\frac{2 t-s}{2 s(t-s)}\right)\right] d r d \theta=M_{211}+M_{212}
\end{aligned}
$$


and

$$
M_{211}=16 \pi\left(\frac{r(A)(t-s)}{2 t-s}\right)^{4}
$$

Shifting we get

$$
\begin{aligned}
M_{212} & =2 \int_{0}^{2 \pi} \int_{0}^{\infty}\left(r+\frac{2 r(A)(t-s)}{2 t-s}\right)^{3} \exp \left[-\frac{r^{2}}{2}\left(\frac{2 t-s}{s(t-s)}\right)\right] d r d \theta \\
& <32 \pi[r(A) \vee 1]\left(\frac{s(t-s)}{2 t-s}\right)^{2}+\frac{80 \pi[r(A) \vee 1]^{3} s(t-s)^{3}}{(2 t-s)^{3}}
\end{aligned}
$$

Similarly,

$$
M_{22}<\frac{2(a s)^{2}}{(2 t-s)^{2}}\left[\frac{2 \pi s(t-s)}{2 t-s}-4 \pi r(A) \sqrt{\frac{(t-s)^{3} s}{(2 t-s)^{3}}}\right]^{+} .
$$

Combining these estimates we have thus shown

$$
\begin{aligned}
& \bar{E}_{a}\left[\left(N_{A}(t)\right)^{n}\right]>\frac{(m(A))^{n}(\tilde{n})^{n-1} n !}{(2 \pi)^{n} 4^{n-1} \cdot 2} \exp \left[-\frac{\left(a-c_{a}^{\prime}\right)^{2}}{2 t}\right] \\
& \cdot \sum_{k=1}^{n-1}\left\{\int_{1}^{t} \frac{(\ln s)^{n-2}}{2 \pi(t-s) s^{2}}\left(1-\frac{\left(a-c_{a}^{\prime}\right)^{2} s}{2 t(2 t-s)}\right)^{+}\left(1-\frac{Q_{1}(A, n, k)}{\ln s}\right)^{+}\right. \\
& \cdot\left[\frac{2 \pi s(t-s)}{2 t-s}-\frac{4 \pi r(A)(t-s)^{3 / 2}(\pi s)^{1 / 2}}{(2 t-s)^{3 / 2}}\right]^{+} d s \\
& -c(n, k) \int_{1}^{t} \frac{(\ln s)^{n-3}}{2 \pi(t-s) s^{3}}\left(1-\frac{\left(a-c_{a}^{\prime}\right)^{2} s}{2 t(2 t-s)}\right)^{+} \\
& \cdot\left[\frac{48 \pi[r(A) \vee 1]^{4} s^{2}(t-s)^{2}}{(2 t-s)^{2}}+\frac{80 \pi[r(A) \vee 1]^{3} s(t-s)^{3}}{(2 t-s)^{3}}\right. \\
& \left.\left.+\frac{4 \pi a^{2} s^{3}(t-s)}{(2 t-s)^{3}}\right] d s\right\} \\
& =\frac{(m(A))^{n}(\tilde{n})^{n-1} n !}{(2 \pi)^{n} 4^{n-1} \cdot 2} \exp \left[-\frac{\left(a-c_{a}^{\prime}\right)^{2}}{2 t}\right]_{k=1}^{n-1}\left(L_{1}+L_{2}\right)
\end{aligned}
$$


where $c(n, k)=c(k)+c(n-k)$ and $Q_{1}(A, n, k)=\Omega_{l}(A, k)+\Omega_{l}(A, n-$ $k)$. We can bound $L_{1}$ from below by

$$
\begin{gathered}
\int_{1}^{t} \frac{(\ln s)^{n-2}}{s(2 t-s)} d s-\int_{1}^{t} \frac{(\ln s)^{n-2}\left(a-c_{a}^{\prime}\right)^{2}}{2 t(2 t-s)^{2}} d s-\int_{1}^{t} \frac{(\ln s)^{n-3} Q_{1}(A, n, k)}{s(2 t-s)} d s \\
\left.-\int_{1}^{t} \frac{2 \pi^{1 / 2} r(A) s^{1 / 2}(\ln s)^{n-2}(t-s)^{1 / 2}}{s^{2}(2 t-s)^{3 / 2}} d s\right] .
\end{gathered}
$$

Since $\left|c_{a}^{\prime}\right| \leqslant r(A)$ and $\ln s \leqslant 2 s^{1 / 2}$ for $s \geqslant 1$ we have

$$
\begin{aligned}
L_{1} \geqslant & \frac{(\ln t)^{n-1}}{2(n-1) t}-\frac{(\ln t)^{n-2} \cdot a^{2}}{2 t^{2}}-\frac{(r(A))^{2}}{2(n-2)} \frac{(\ln t)^{n-2}}{t} \\
& -\frac{Q_{1}(A, n, k)(\ln t)^{n-2}}{(n-2) t}-\frac{4 r(A) \sqrt{\pi}}{n-2} \frac{(\ln t)^{n-2}}{t} .
\end{aligned}
$$

We can bound $L_{2}$ from below by

$$
\begin{array}{r}
-c(n, k) \int_{1}^{t} \frac{(\ln s)^{n-3}}{2 \pi(t-s) s^{3}}\left[\frac{48 \pi[r(A) \vee 1]^{4} s^{2}(t-s)^{2}}{(2 t-s)^{2}}\right. \\
\left.\quad+\frac{80 \pi[r(A) \vee 1]^{3} s(t-s)^{3}}{(2 t-s)^{3}}+\frac{4 \pi a^{2} s^{3}(t-s)}{(2 t-s)^{3}}\right] \\
>-c(n, k) \frac{(\ln s)^{n-2}}{(n-2) t}\left(24 \pi[r(A) \vee 1]^{4}+40 \pi[r(A) \vee 1]^{3}+\frac{a^{2}(\ln t)^{n-2}}{(n-2) t}\right) .
\end{array}
$$

Thus adding everything together we get

$$
\begin{aligned}
\bar{E}_{a}\left[\left(N_{A}(t)\right)^{n}\right]>\exp \left[-\frac{\left(a-c_{a}^{\prime}\right)^{2}}{2 t}\right] & \cdot \frac{(m(A))^{n}(\tilde{n})^{n-1} n !}{(2 \pi)^{n} 4^{n-2} 2} \chi_{(t>1)} \\
\cdot \sum_{k=1}^{n-1}\left\{\frac{(\ln t)^{n-1}}{2(n-1) t}-\frac{(\ln t)^{n-2}}{2(n-2) t}[\right. & 2 Q_{1}(A, n, k)+(r(A))^{2}+8 \pi^{1 / 2} r(A) \\
& \left.+c(n, k)\left(48[r(A) \vee 1]^{4}+80[r(A) \vee 1]^{3}\right)\right] \\
& \left.-\frac{(\ln t)^{n-2} a^{2}}{2(n-1) t^{2}}[2 c(n, k)+n-1]\right\}^{+} .
\end{aligned}
$$

Now, since $c(n, k)=c(k)+c(n-k), c(k)=6^{k-1}$ and $n-1<6^{n-2}$ for $n>3$, we get 


$$
\sum_{k=1}^{n-1} \frac{(\ln t)^{n-2} a^{2}}{2(n-1) t^{2}}[2 c(n, k)+(n-1)]<\frac{c(n) a^{2}(\ln t)^{n-1}}{2 t \ln t} .
$$

Lastly, since by definition $Q_{1}(A, n, k)=\Omega_{l}(A, k)+\Omega_{l}(A, n-k)$ with $\Omega_{l}(A, k)=\left(32[r(A) \vee 1]^{2}\right)^{k-1}$ we obtain

$$
\begin{gathered}
\sum_{k=1}^{n-1}\left\{\frac{2 Q_{1}(A, n, k)}{n-2}+\frac{31\left[(r(A))^{2} \vee 1\right]}{n-1}+\frac{128\left[(r(A))^{4} \vee 1\right] c(n, k)}{n-2}\right\} \\
<\Omega_{l}(A, n)
\end{gathered}
$$

which finally shows

$$
\begin{aligned}
\bar{E}_{a}\left[\left(N_{A}(t)\right)^{n}\right]> & \exp \left[-\frac{\left(a-c_{a}^{\prime}\right)^{2}}{2 t}\right] \\
& \cdot \frac{(m(A))^{n}(\tilde{n})^{n-1} n !}{(2 \pi)^{n} 4^{n-1}} \chi_{(t>1)} \frac{(\ln t)^{n-1}}{t}\left(1-\frac{c(n) a^{2}}{t \ln t}-\frac{\Omega_{l}(A, n)}{\ln t}\right)^{+}
\end{aligned}
$$

thus proving the lemma.

Next we wish to obtain an upper bound on $\bar{E}_{a}\left(N_{A}(t)\right)^{n}$. Therefore,

LEMMA 2.3.

$$
\begin{aligned}
\bar{E}_{a}\left[\left(N_{A}(t)\right)^{k}\right]<c(A, k) \exp \left[-\frac{\left(a-c_{a}^{\prime}\right)^{2}}{2 t}\right] \chi_{(t<e)} \\
+\chi(t>e)\left\{\exp \left[-\frac{\left(a-c_{a}\right)^{2}}{2 t}\right] \cdot \frac{k !(m(A))^{k}(\tilde{n})^{k-1}}{4^{k-1}(2 \pi)^{k}} \frac{(\ln t)^{k-1}}{t}\right. \\
\left.+\exp \left[-\frac{\left(a-c_{a}^{\prime}\right)^{2}}{2 t}\right] \cdot \frac{\Omega_{u}(A, k)(\ln t)^{k-2}}{t}\right\}
\end{aligned}
$$

for all $t, k$ positive, and where $\left(a-c_{a}\right)^{2}=\min _{c \in A}(a-c)^{2},\left(a-c_{a}^{\prime}\right)^{2}=$ $\min _{c \in A+r(A)}(a-c)^{2}, \Omega_{u}(A, k)=8^{k}(\tilde{n})^{k-1} k !\left(30\left[(r(A))^{6} \vee 1\right)^{k-1}\right.$ and

$$
c(A, k)= \begin{cases}(2 k-3) ! !(\tilde{n})^{k-1}\left(15\left[(r(A))^{2} \vee 1\right]\right)^{k-1}, & k>1, \\ 1, & k=1 .\end{cases}
$$

ProOF. To simplify the computation we first prove the following lemma:

LEMMA 2.4. Under the same conditions as above, 
$\int_{D} p(t-s, a, b) \exp \left[-\frac{\left(b-c_{b}^{\prime}\right)^{2}}{s}\right] d b$

(1)

$$
\begin{aligned}
& <\exp \left[-\frac{\left(a-c_{a}^{\prime}\right)^{2}}{2 t}\right] \\
& \cdot\left\{\frac{s}{2 t-s}+\frac{8(r(A))^{2}(t-s)}{(2 t-s)^{2}}+\frac{4 \sqrt{2} r(A) s^{1 / 2}(t-s)^{1 / 2}}{(2 t-s)^{3 / 2}}\right\}
\end{aligned}
$$

and

(2)

$$
\begin{aligned}
\int_{D} p(t-s, a, b) \exp \left[-\frac{\left(b-c_{b}\right)^{2}}{s}\right] d b \\
<\exp \left[-\frac{\left(a-c_{a}\right)^{2}}{2 t}\right] \\
\cdot\left\{\frac{s}{2 t-s}+\frac{2(r(A))^{2}(t-s)}{(2 t-s)^{2}}+\frac{\sqrt{2} r(A) s^{1 / 2}(t-s)^{1 / 2}}{(2 t-s)^{3 / 2}}\right\}
\end{aligned}
$$

Proof. We prove (1) as (2) follows by a similar proof.

As before,

$$
\begin{aligned}
\int_{D} p(t-s, a, b) \exp \left[-\frac{\left(b-c_{b}^{\prime}\right)^{2}}{s}\right] d b \\
=\frac{1}{2 \pi(t-s)} \int_{D} \exp \left[-\frac{(a-b)^{2}}{2(t-s)}\right] \cdot \exp \left[-\frac{\left(b-c_{b}^{\prime}\right)^{2}}{s}\right] d b \\
<\exp \left[-\frac{\left(a-c_{a}^{\prime}\right)^{2}}{2 t}\right] \\
\cdot \frac{1}{2 \pi(t-s)} \int_{D} \exp \left[-\left(b-\frac{2 \hat{c}_{b}(t-s)}{2 t-s}\right)^{2}\left(\frac{2 t-s}{2 s(t-s)}\right)\right] d b \\
<\exp \left[-\frac{\left(a-c_{a}^{\prime}\right)^{2}}{2 t}\right] \cdot \frac{1}{2 \pi(t-s)} \\
\quad \cdot\left\{\int_{0}^{2 \pi} \int_{0}^{4 r(A)(t-s) /(2 t-s)} r d r d \theta+\int_{0}^{2 \pi} \int_{4 r(A)(t-s) /(2 t-s)}^{\infty}\right. \\
\left.\quad \cdot \exp \left[-\left(r-\frac{4 r(A)(t-s)}{2 t-s}\right)^{2}\left(\frac{2 t-s}{2 s(t-s)}\right)\right] \cdot r d r d \theta\right\}
\end{aligned}
$$

(1)

since $\left|c_{b}^{\prime}\right|<2 r(A)$ and $\hat{c}_{b} \in A$. Shifting and integrating we see that 
(1) $<\exp \left[-\frac{\left(a-c_{a}^{\prime}\right)^{2}}{2 t}\right]\left\{\frac{s}{2 t-s}+\frac{8(r(A))^{2}(t-s)}{(2 t-s)^{2}}+\frac{4 \sqrt{2} r(A) s^{1 / 2}(t-s)^{1 / 2}}{(2 t-s)^{3 / 2}}\right\}$,

thus verifying Lemma 2.4 .

Now, returning to the proof of Lemma 2.3 which we again prove by induction. For $k=1$,

$$
\begin{aligned}
\bar{E}_{a}\left(N_{A}(t)\right)= & \int_{A} p(t, a, b) d b \\
= & \chi_{(t<e)} \cdot \frac{1}{2 \pi t} \int_{A} \exp \left[-\frac{(a-b)^{2}}{2 t}\right] d b \\
& +\chi_{(t>e)} \int_{A} \frac{1}{2 \pi t} \exp \left[-\frac{(a-b)^{2}}{2 t}\right] d b \\
= & J_{1}+J_{2} .
\end{aligned}
$$

We must show

$$
\exp \left[-\frac{\left(a-c_{a}^{\prime}\right)^{2}}{2 t}\right] \cdot \chi_{(t<e)} \geqslant J_{1} \text {. }
$$

However, since $p(t, a, b) \geqslant 0$ for all $t, a, b$ and $\int_{D} p(t, a, b) d b=1$, and since for $a \in A+r(A), \exp \left[-\left(a-c_{a}^{\prime}\right)^{2} / 2 t\right] \cdot \chi_{(t<e)} \geqslant \chi_{(t<e)}$ we must just verify (2) for $a \notin A+r(A)$. However then $\left|a-c_{a}\right| \geqslant r(A)$ which implies

$$
\exp \left[-\frac{\left(a-c_{a}^{\prime}\right)^{2}}{2 t}\right] \geqslant \frac{(r(A))^{2}}{2 t} \exp \left[-\frac{\left(a-c_{a}\right)^{2}}{2 t}\right]
$$

Thus, since $m(A) \leqslant \pi(r(A))^{2}$, (2) holds for all $a$. Observing that

$$
J_{2} \leqslant \chi_{(t>e)} \frac{m(A)}{2 \pi t} \exp \left[-\frac{\left(a-c_{a}\right)^{2}}{2 t}\right],
$$

we see that the lemma holds for $k=1$.

Now, assume the lemma correct for $k<n$ then by the definition of $\bar{E}_{a}\left(N_{A}(t)\right)^{n}$ and the induction hypothesis we have

$$
\begin{aligned}
\bar{E}_{a}\left[\left(N_{A}(t)\right)^{n}\right] \leqslant \frac{\tilde{n}}{2} \sum_{k=1}^{n-1}\left(\begin{array}{l}
n \\
k
\end{array}\right) \\
\quad\left\{\begin{array}{l}
\int_{0}^{t \wedge e} c(A, k) c(A, n-k) \\
\cdot\left[\int_{D} p(t-s, a, b) \exp \left[-\frac{\left(b-c_{b}^{\prime}\right)^{2}}{s}\right] d b\right] d s
\end{array}\right.
\end{aligned}
$$




$$
\begin{gathered}
+\chi_{(t>e)} \int_{e}^{t} \frac{k !(n-k) !(m(A))^{n}(\tilde{n})^{n-2}}{(2 \pi)^{n} 4^{n-2}} \frac{(\ln s)^{n-2}}{s^{2}} \\
\cdot\left[\int_{D} p(t-s, a, b) \exp \left[-\frac{\left(b-c_{b}\right)^{2}}{s}\right] d b\right] d s \\
+\chi_{(t>e)} \int_{e}^{t} \Omega_{u}(A, k, n-k) \frac{(\ln s)^{n-3}}{s^{2}} \\
\left.\cdot\left[\int_{D} p(t-s, a, b) \exp \left[-\frac{\left(b-c_{b}^{\prime}\right)^{2}}{s}\right] d b\right] d s\right\}
\end{gathered}
$$

where

$$
\begin{aligned}
\Omega_{u}(A, k, n-k)= & \Omega_{u}(A, k) \Omega_{u}(A, n-k) \\
& +\Omega_{u}(A, k) \frac{(n-k) !(m(A))^{n-k}(\tilde{n})^{n-k-1}}{(2 \pi)^{n-k} 4^{n-k-2}} \\
& +\Omega_{u}(A, n-k) \frac{k !(m(A))^{k}(\tilde{n})^{k-1}}{(2 \pi)^{k} 4^{k-2}} .
\end{aligned}
$$

Applying Lemma 2.4 gives

$$
\begin{aligned}
& \bar{E}_{a}\left[\left(N_{A}(t)\right)^{n}\right]<\frac{\tilde{n}}{2} \sum_{k=1}^{n-1}\left(\begin{array}{l}
n \\
k
\end{array}\right) \\
& \left\{c(A, k) c(A, n-k) \chi_{(t<e)} \cdot \exp \left[-\frac{\left(a-c_{a}^{\prime}\right)^{2}}{2 t}\right]\right. \\
& \cdot \int_{0}^{t}\left[\frac{s}{2 t-s}+\frac{8(r(A))^{2}(t-s)}{(2 t-s)^{2}}+\frac{4 \sqrt{2} r(A) s^{1 / 2}(t-s)^{1 / 2}}{(2 t-s)^{3 / 2}}\right] d s \\
& +c(A, k) c(A, n-k) \chi_{(t>e)} \cdot \exp \left[-\frac{\left(a-c_{a}^{\prime}\right)^{2}}{2 t}\right] \\
& \cdot \int_{0}^{e}\left[\frac{s}{2 t-s}+\frac{8(r(A))^{2}(t-s)}{(2 t-s)^{2}}+\frac{4 \sqrt{2} r(A) s^{1 / 2}(t-s)^{1 / 2}}{(2 t-s)^{3 / 2}}\right] d s \\
& +\frac{k !(n-k) !(m(A))^{n}(\tilde{n})^{n-2}}{(2 \pi)^{n} 4^{n-2}} \chi_{(t>e)} \exp \left[-\frac{\left(a-c_{a}\right)^{2}}{2 t}\right]
\end{aligned}
$$




$$
\begin{gathered}
\int_{e}^{t} \frac{(\ln s)^{n-2}}{s^{2}}\left[\frac{s}{2 t-s}+\frac{(2 r(A))^{2}(t-s)}{(2 t-s)^{2}}+\frac{\sqrt{2} r(A) s^{1 / 2}(t-s)^{1 / 2}}{(2 t-s)^{3 / 2}}\right] d s \\
+\Omega_{u}(A, k, n-k) \chi_{(t>e)} \exp \left[-\frac{\left(a-c_{a}^{\prime}\right)^{2}}{2 t}\right] \\
\cdot \int_{e}^{t} \frac{(\ln s)^{n-3}}{s^{2}}\left[\frac{s}{2 t-s}+\frac{8(r(A))^{2}(t-s)}{(2 t-s)^{2}}\right. \\
=\frac{\tilde{n}}{2} \sum_{k=1}^{n-1}\left(\begin{array}{l}
n \\
k
\end{array}\right)\left(L_{1}(k)+L_{2}(k)+L_{3}(k)+L_{4}(k)\right) .
\end{gathered}
$$

For $L_{1}$ we use

$$
\int_{0}^{t} \frac{t-s}{(2 t-s)^{2}} d s=\ln 2-\frac{1}{2}, \quad \int_{0}^{t} \frac{(t-s)^{1 / 2}}{(2 t-s)^{3 / 2}} d s \leqslant 2-\sqrt{2},
$$

and

$$
\int_{0}^{t} \frac{s}{2 t-s} d s<2
$$

Thus,

$L_{1}<c(A, k) c(A, n-k) \chi_{(t<e)} \exp \left[-\frac{\left(a-c_{a}^{\prime}\right)^{2}}{2 t}\right]\left(2+3(r(A))^{2}+10 r(A)\right)$.

For $L_{2}$, using $1 /(2 t-s) \leqslant 1 / t$ and $(t-s) /(2 t-s)<1$ we obtain

$$
\begin{aligned}
L_{2} \leqslant & c(A, k) c(A, n-k) \chi_{(t>e)} \exp \left[-\frac{\left(a-c_{a}^{\prime}\right)^{2}}{2 t}\right] \\
& \cdot \frac{1}{t}\left(\frac{e^{2}}{2}+8(r(A))^{2}+\frac{8 e}{3} r(A) \sqrt{2 e}\right) .
\end{aligned}
$$

Using the obvious inequalities, $\ln s / \sqrt{s} \leq \sqrt{e}$ and $\ln s / s<e$ for $s>e$ and observing that

$$
\frac{1}{2 t} \int_{e}^{t} \frac{(\ln s)^{n-2}}{s(1-s / 2 t)} d s<\frac{(\ln t)^{n-1}}{2(n-1) t}+\frac{(\ln t)^{n-2}}{2 t}
$$

we obtain 


$$
\begin{aligned}
L_{3} \leqslant & \chi_{(t>e)} \cdot \exp \left[-\frac{\left(a-c_{a}\right)^{2}}{2 t}\right] \cdot \frac{k !(n-k) !(m(A))^{n}(\tilde{n})^{n-2}}{(2 \pi)^{n} 4^{n-2}} \\
& \cdot\left\{\frac{(\ln t)^{n-1}}{2(n-1) t}+\frac{(\ln t)^{n-2}}{t}\left[\frac{1}{2}+\frac{2(r(A))^{2}}{(n-2) e}+\frac{\sqrt{2} r(A)}{\sqrt{e}(n-2)}\right]\right\} .
\end{aligned}
$$

Lastly,

$$
\begin{aligned}
L_{4} & <\Omega_{u}(A, k, n-k) \chi_{(t>e)} \\
& \cdot \exp \left[-\frac{\left(a-c_{a}^{\prime}\right)^{2}}{2 t}\right] \frac{\left[(\ln t)^{n-2}-1\right]}{(n-2) t}\left[1+\frac{4 \sqrt{2}}{\sqrt{e}} r(A)+\frac{8(r(A))^{2}}{e}\right) .
\end{aligned}
$$

In order to evaluate the sum we need the following result:

LEMMA 2.5. If

$$
l(n)=\frac{1}{2} \sum_{k=1}^{n-1}\left(\begin{array}{l}
n \\
k
\end{array}\right) l(k) l(n-k) \text { for } n>1, l(1)=1, l(0)=0
$$

then $l(n)=(2 n-3) ! !$ for $n>1$.

Proof. Letting $\hat{l}(s)$ be the exponential generating function of $l(n)$ we have

$$
\begin{aligned}
\hat{l}(s) & =\sum_{n>0} \frac{l(n) s^{n}}{n !}=s+\frac{1}{2} \sum_{n=0}^{\infty} \sum_{k=1}^{\infty} \frac{l(k) s^{k}}{k !} \frac{l(n-k)}{(n-k) !} s^{n-k} \\
& =s+\frac{1}{2}(\hat{l}(s))^{2} .
\end{aligned}
$$

Since $\hat{l}(0)=0$ we have $\hat{l}(s)=1-\sqrt{1-2 s}$. Expanding $\sqrt{1-2 s}$ in a Taylor series and comparing coefficients completes the proof.

Thus,

$$
\frac{\tilde{n}}{2} \sum_{k=1}^{n-1}\left(\begin{array}{l}
n \\
k
\end{array}\right) L_{1}(k)<(2 n-3) ! !(\tilde{n})^{n-1}\left(15\left[(r(A))^{2} \vee 1\right]\right)^{n-1}
$$

$$
\begin{aligned}
& \cdot \chi_{(t<e)} \cdot \exp \left[-\frac{\left(a-c_{a}^{\prime}\right)^{2}}{2 t}\right] \\
& \frac{\tilde{n}}{2} \sum_{k=1}^{n-1}\left(\begin{array}{l}
n \\
k
\end{array}\right) L_{2}(k)<(2 n-3) ! !(\tilde{n})^{n-1} \chi_{(\ell>e)} \\
& \cdot \frac{4}{t}\left(15\left[(r(A))^{2} \vee 1\right]\right)^{n-1} \cdot \exp \left[-\frac{\left(a-c_{a}^{\prime}\right)^{2}}{2 t}\right]
\end{aligned}
$$


(6)

$$
\begin{aligned}
\frac{\tilde{n}}{2} \sum_{k=1}^{n-1}\left(\begin{array}{l}
n \\
k
\end{array}\right) L_{3}(k) \leqslant \chi_{(t>e)} \cdot \exp \left[-\frac{\left(a-c_{a}\right)^{2}}{2 t}\right] & \left\{\frac{(\ln t)^{n-1}(\tilde{n})^{n-1}(m(A))^{n} n !}{(2 \pi)^{n} 4^{n-1} t}\right. \\
& \left.+\frac{(\ln t)^{n-2} \cdot n !(r(A))^{2 n}(\tilde{n})^{n-1}}{t \cdot 8^{n-1}}\left(\frac{n-1}{2}+\frac{n-1}{n-2} 2\left[(r(A))^{2} \vee 1\right]\right)\right\}
\end{aligned}
$$

By the definition of $\Omega_{u}(A, k, n-k)$ we get

$$
\begin{aligned}
\frac{\tilde{n}}{2} \sum_{k=1}^{n-1}\left(\begin{array}{l}
n \\
k
\end{array}\right) & L_{4}(k)<\chi_{(t>e)} \cdot(\tilde{n})^{n-1} \exp \left[-\frac{\left(a-c_{a}^{\prime}\right)^{2}}{2 t}\right] \\
& \frac{\left[(\ln t)^{n-1}-1\right] 8\left[(r(A))^{2} \vee 1\right]}{2(n-2) t} \\
& \cdot \sum_{k=1}^{n-1}\left(\begin{array}{l}
n \\
k
\end{array}\right)\left\{(n-k) ! k ! 8^{n}\left(30\left[(r(A))^{6} \vee 1\right]\right)^{n-2}\right. \\
+ & \left.\frac{2(n-k) ! k ! 8^{k}(m(A))^{n-k}\left(30\left[(r(A))^{6} \vee 1\right]\right)^{k-1}}{(2 \pi)^{n-k} 4^{n-k-1}}\right\} .
\end{aligned}
$$

However, since

$$
\begin{gathered}
\sum_{k=1}^{n-1}\left(\begin{array}{l}
n \\
k
\end{array}\right) \frac{2(n-k) ! k ! 8^{k}\left(30\left[(r(A))^{6} \vee 1\right]\right)^{k-1}(m(A))^{n-k}}{(2 \pi)^{n-k} 4^{n-k-1}} \\
\leqslant n !\left[(r(A))^{6} \vee 1\right]^{n-2} \frac{8^{n}(30)^{n-1}}{239}
\end{gathered}
$$

we get for $n>2$,

$$
\frac{\tilde{n}}{2} \sum_{k=1}^{n-1}\left(\begin{array}{l}
n \\
k
\end{array}\right) L_{4}(k) \leqslant n ! \chi_{(t>e)} \cdot \exp \left[-\frac{\left(a-c_{a}^{\prime}\right)^{2}}{2 t}\right]
$$

$$
\begin{aligned}
& \cdot(\tilde{n})^{n-1} 8^{n}\left(30\left[(r(A))^{6} \vee 1\right]\right)^{n-1} \\
& \cdot\left[\frac{8(n-1)}{60(n-2)}+\frac{1}{58(n-2)}\right]\left(\frac{(\ln t)^{n-2}-1}{t}\right) .
\end{aligned}
$$


Now using $(2 n-3) ! ! \leqslant\left(2^{n} \cdot n !\right) /(2 n-1) \sqrt{\pi n}$ for $n>2$ and noticing that the $L_{2}(k)$ contribution is cancelled off by the $-1 / t$ term from $L_{4}$ we get using (3), (4), (5), (6), and (7) for $n>2$,

$$
\begin{aligned}
& \bar{E}_{a}\left[\left(N_{A}(t)\right)^{n}\right] \leqslant \chi_{(t<e)}(2 n-3) ! !\left(15\left[(r(A))^{2} \vee 1\right]\right)^{n-1} \cdot \exp \left[-\frac{\left(a-c_{a}^{\prime}\right)^{2}}{2 t}\right] \\
& \quad+\chi_{(t>e)} \frac{(\ln t)^{n-1}}{t} \frac{n !(\tilde{n})^{n-1}(m(A))^{n}}{(2 \pi)^{n} 4^{n-1}} \exp \left[-\frac{\left(a-c_{a}\right)^{2}}{2 t}\right] \\
& \quad+\chi_{(t>e)} \frac{(\ln t)^{n-2}}{t} n !(\tilde{n})^{n-1} 8^{n}\left(30\left[(r(A))^{6} \vee 1\right]\right)^{n-1} \exp \left[-\frac{\left(a-c_{a}^{\prime}\right)^{2}}{2 t}\right]
\end{aligned}
$$

A simple calculation shows the validity for $n=2$ thus proving Lemma 2.3.

For purposes of calculation it will be convenient to have the following weaker bound on $\bar{E}_{a}\left[\left(N_{A}(t)\right)^{n}\right]$ :

Corollary 2.6.

$$
\begin{aligned}
& \bar{E}_{a}\left[\left(N_{A}(t)\right)^{n}\right] \leqslant \chi_{(t<e)}(2 n-3) ! !\left(15\left[(r(A))^{2} \vee 1\right]\right)^{n-1} \exp \left[-\frac{\left(a-c_{a}^{\prime}\right)^{2}}{2 t}\right] \\
& \quad+2 \chi_{(t>e)} \frac{(\ln t)^{n-1}}{t} n !(\tilde{n})^{n-1} 8^{n}\left(30\left[(r(A))^{6} \vee 1\right]\right)^{n-1} \exp \left[-\frac{\left(a-c_{a}^{\prime}\right)^{2}}{2 t}\right]
\end{aligned}
$$

Now to determine $E_{a}\left[\left(N_{A}(t)\right)^{n}\right]$, we use Lemma 1.1, Definition 2.1 and set $\tilde{E}_{a}\left[\left(N_{A}(t)\right)^{k}\right]=E_{a}\left[\left(N_{A}(t)\right)^{k}\right]-\bar{E}_{a}\left[\left(N_{A}(t)\right)^{k}\right]$ then:

LEMMA 2.7.

$$
\begin{aligned}
\bar{E}_{a}\left[\left(N_{A}(t)\right)^{k}\right] \leqslant E_{a}\left[\left(N_{A}(t)\right)^{k}\right] \\
=\int_{A} p(t, a, b) d b+\bar{E}_{a}\left[\left(N_{A}(t)\right)^{k}\right]+\sum_{l=2}^{k} \frac{\tilde{n}_{l}}{l !} \sum_{\left(j_{1}, v_{l}\right)^{ \pm}} \frac{k !}{j_{1} ! \cdots j_{l} !} \\
\quad \cdot \sum_{\left(\varepsilon_{1}, \varepsilon_{l}\right)^{*}} \int_{0}^{ \pm} \int_{D} p(t-s, a, b) E_{b}^{\varepsilon_{1}}\left[\left(N_{A}(s)\right)^{j_{1}}\right] \cdots E_{b^{\varepsilon_{l}}}\left[\left(N_{A}(s)\right)^{j_{l}}\right] d b d s
\end{aligned}
$$

where $\varepsilon_{i}=0,1$ with $E_{b}^{0}=\tilde{E}_{b}$ and $E_{b}^{1}=\bar{E}_{b}$, where $\Sigma_{\left(j_{1}, j_{j} \pm\right.}$ denotes summation over all ways of partitioning $k$ into $l$ positive integers and $\Sigma_{\left(\varepsilon_{1}, e_{1}\right)^{*}}$ signifies summation over all combinations of $\varepsilon_{1},, \varepsilon_{l}$ except that for $l=2$ the term $\varepsilon_{1}=\varepsilon_{2}=1$ is left out.

REMARK 2.8 . 


$$
\sum_{\left(\varepsilon_{1}, \varepsilon_{l}\right)^{*}} 1= \begin{cases}2^{l}, & l \neq 2 \\ 3, & l=2,\end{cases}
$$

since $\varepsilon_{i}=0,1$ except for $l=2$.

LEMMA 2.9.

$$
\begin{aligned}
E_{a}\left[\left(N_{A}(t)\right)^{n}\right]< & \bar{E}_{a}\left[\left(N_{A}(t)\right)^{n}\right]+C_{1}(A, n) l^{\prime}(n) \chi_{(\ell<e)} \cdot \exp \left[-\frac{\left(a-c_{a}^{\prime}\right)^{2}}{2 t}\right] \\
& +C_{2}(A, n) l^{\prime}(n) \chi_{(t>e)} \frac{(\ln t)^{n-2}}{t} \exp \left[-\frac{\left(a-c_{a}^{\prime}\right)^{2}}{2 t}\right]
\end{aligned}
$$

where $\sum_{n=0}^{\infty}\left(l^{\prime}(n) z^{n} / n !\right)=f(z)$ is a solution of $z=1+2 f(z)-e^{f(z)}$, $C_{1}(A, n)<\left(60\left[(r(A))^{2} \vee 1\right] 2 C^{2}\right)^{n-1}, C_{2}(A, n)<\left[120\left[(r(A))^{6} \vee 1\right] \cdot\left(2 C^{2}\right)\right]^{n-1}$ where $C$ is chosen so that $\tilde{n}_{r} \leqslant C^{r}(C \geqslant 1)$.

Proof. Again by induction; for $n=1$ the lemma is obvious since $E_{a}\left(N_{A}(t)\right)=\bar{E}_{a}\left(N_{A}(t)\right)$. For $n=2$,

$$
\begin{aligned}
E_{a}\left[\left(N_{A}(t)\right)^{2}\right] & -\bar{E}_{a}\left[\left(N_{A}(t)\right)^{2}\right]=\int_{A} p(t, a, b) d b \\
& <\chi_{(t<e)} \exp \left[-\frac{\left(a-c_{a}^{\prime}\right)^{2}}{2 t}\right]+\chi_{(t>e)} \frac{m(A)}{2 \pi t} \exp \left[-\frac{\left(a-c_{a}\right)^{2}}{2 t}\right]
\end{aligned}
$$

(as in the case $k=1$ of Lemma 2.3). Assume the lemma proven for $k<n$. By Lemma 2.7 if we let

$$
M_{1}=\int_{A} p(t, a, b) d b
$$

and

$$
\begin{aligned}
M_{2} & =\sum_{l=2}^{n} \frac{\tilde{n}_{l}}{l !} \sum_{\left(j_{1}, j_{l}\right)^{ \pm}} \frac{n !}{j_{1} ! \cdots j_{l} !} \\
& \cdot \sum_{\left(\varepsilon_{1}, \varepsilon_{l}\right)^{*}} \int_{0}^{t} \int_{D} p(t-s, a, b) E_{b}^{\varepsilon_{1}}\left[\left(N_{A}(s)\right)^{j_{1}}\right] \cdots E_{b}^{\varepsilon^{\varepsilon}}\left[\left(N_{A}(s)\right)^{j_{l}}\right] d b d s
\end{aligned}
$$

then $E_{a}\left[\left(N_{A}(t)\right)^{n}\right]-\bar{E}_{a}\left[\left(N_{A}(t)\right)^{n}\right]=M_{1}+M_{2}$.

Again,

$$
M_{1}<\chi_{(t<e)} \exp \left[-\frac{\left(a-c_{a}^{\prime}\right)^{2}}{2 t}\right]+\chi_{(t>e)} \frac{m(A)}{2 \pi t} \exp \left[-\frac{\left(a-c_{a}\right)^{2}}{2 t}\right] .
$$

Using Corollary 2.6 and since $(2 k-3) ! !\left(15\left[(r(A))^{2} \vee 1\right]\right)^{k-1} \leqslant$ $l^{\prime}(k)\left(60\left[(r(A))^{2} \vee 1\right]\right)^{k-1}$ we get 


$$
\begin{aligned}
M_{2}<\sum_{l=2}^{n} \frac{\tilde{n}_{l}}{l !} \sum_{\left(j_{1}, j_{l}\right)^{ \pm}} \frac{n ! l^{\prime}\left(j_{1}\right) \cdots l^{\prime}\left(j_{l}\right)}{j_{1} ! \cdots j_{l} !} & \sum_{\left(\varepsilon_{1}, \ldots, \varepsilon_{l}\right)^{*}}\left\{\left(60\left[(r(A))^{2} \vee 1\right]\right)^{n-l}\left(2 C^{2}\right)^{n-l}\right. \\
& \cdot \int_{0}^{t \wedge e} \int_{D} p(t-s, a, b) \exp \left[-\frac{\left(b-c_{b}^{\prime}\right)^{2}}{s}\right] d b d s \\
& +\left(120\left[(r(A))^{6} \vee 1\right]\right)^{n-l}\left(2 C^{2}\right)^{n-l} \chi(t>e) \int_{e}^{t} \frac{(\ln s)^{n+j-2 l}}{s^{l}} \\
& \left.\cdot\left[\int_{D} p(t-s, a, b) \exp \left[-\frac{\left(b-c_{b}^{\prime}\right)^{2}}{s}\right] d b\right] d s\right\}
\end{aligned}
$$

where $j=\sum_{i=1}^{l} \varepsilon_{i}$.

As we showed in proving Lemma 2.3,

$$
\begin{aligned}
& \int_{0}^{t \wedge e} \int_{D} p(t-s, a, b) \exp \left[-\frac{\left(b-c_{b}^{\prime}\right)^{2}}{s}\right] d b d s \\
& <\chi_{(t<e)} \cdot \exp \left[-\frac{\left(a-c_{a}^{\prime}\right)^{2}}{2 t}\right] 15\left[(r(A))^{2} \vee 1\right] \\
& +\chi_{(t>e)} \exp \left[-\frac{\left(a-c_{a}^{\prime}\right)^{2}}{2 t}\right] \cdot \frac{45\left[(r(A))^{2} \vee 1\right]}{t} .
\end{aligned}
$$

Now, since $2 l-j \geqslant 3$ (since $l \geqslant 2$ and for $l=2, j<2$ ) by Lemma 2.4 we obtain,

$$
\begin{gathered}
\chi_{(t>e))} \int_{e}^{t} \frac{(\ln s)^{n+j-2 l}}{s^{l}}\left[\int_{D} p(t-s, a, b) \exp \left[-\frac{\left(b-c_{b}^{\prime}\right)^{2}}{s}\right] d b\right] d s \\
<\chi_{(t>e)} \frac{\left[(\ln t)^{n-2}-1\right]}{(n-2) t}\left[1+\frac{4 \sqrt{2}}{\sqrt{e}} r(A)+\frac{8}{e}(r(A))^{2}\right] \\
\cdot \exp \left[-\frac{\left(a-c_{a}^{\prime}\right)^{2}}{2 t}\right] \quad \text { for } n>2 .
\end{gathered}
$$

Remembering Remark 2.8 and since $\tilde{n}_{l}<C^{l}$, if we add $M_{1}$ and $M_{2}$ we obtain 


$$
\begin{aligned}
& E_{a}\left[\left(N_{A}(t)\right)^{n}\right]-\bar{E}_{a}\left[\left(N_{A}(t)\right)^{n}\right] \\
& \leqslant 2^{n-l}\left(C^{2}\right)^{n-1} \sum_{l=2}^{n} \frac{1}{l !} \sum_{\left(j_{1}, \ldots, j_{l}\right)^{ \pm}} \frac{n ! l^{\prime}\left(j_{1}\right) \cdots l^{\prime}\left(j_{l}\right)}{j_{1} ! \cdots j_{l} !} \\
& \quad \cdot\left\{\chi_{(t<e)} \cdot\left(60\left[(r(A))^{2} \vee 1\right]\right)^{n-1} \cdot \exp \left[-\frac{\left(a-c_{a}^{\prime}\right)^{2}}{2 t}\right]\right. \\
& \left.\quad+\frac{(\ln t)^{n-2}}{t} \chi_{(t>e)} \cdot\left(120\left[(r(A))^{6} \vee 1\right]\right)^{n-1} \cdot \exp \left[-\frac{\left(a-c_{a}^{\prime}\right)^{2}}{2 t}\right]\right\} .
\end{aligned}
$$

In order to evaluate the sum we let

$$
l^{\prime}(n)=\sum_{l=2}^{n} \frac{1}{l !} \sum_{\left(j_{1}, \ldots, j_{l}\right)^{ \pm}} \frac{n ! l^{\prime}\left(j_{1}\right) \cdots l^{\prime}\left(j_{l}\right)}{j_{1} ! \cdots j_{l} !}
$$

and wish to show that if $f(z)=\sum_{n \geqslant 0}\left(l^{\prime}(n) / n !\right) z^{n}$, the exponential generating function for $l^{\prime}(n)$, then $z=1+2 f(z)-e^{f(z)}$.

Therefore, we let $w=\Sigma_{n>0}\left(l^{\prime}(n) z^{n} / n !\right)$ with $l^{\prime}(0)=0, l^{\prime}(1)=1$ be defined as a formal power series in $z$. Comparing coefficients yields $z=w-$ $w^{2} / 2 !-w^{3} / 3 !-\cdots=1+2 w-e^{w}$. However, since $g(w)=1+2 w-e^{w}$ is holomorphic in the unit disc, $g(0)=0, g^{\prime}(0)=1$, by Bloch's theorem $\Sigma_{n>0}\left(l^{\prime}(n) z^{n} / n !\right)<\infty$ where $|z|<\rho$ for some $\rho>0$. Using the complexinversion formula,

$$
\frac{l^{\prime}(n)}{n !}=\frac{1}{2 \pi i n} \int_{C} \frac{d z}{\left(1+2 z-e^{z}\right)^{n}}
$$

where $C$ is some closed contour containing the singularity at the origin and no other singularity. Therefore $l^{\prime}(n) \leqslant n !\left(c_{1}\right)^{n}$ for all $n$ completing the proof.

Combining Lemmas 2.2, 2.3, 2.7 and 2.9 we have thus shown,

Corollary 2.10 .

$$
E\left[\left(N_{A}(t)\right)^{n}\right]=\frac{n !(\tilde{n})^{n-1}(m(A))^{n}}{4^{n-1}(2 \pi)^{n}} \frac{(\ln t)^{n-1}}{t}+O\left(\frac{c^{n} n !(\ln t)^{n-2}}{t}\right)
$$

for all positive $n$, for $t \geqslant e, a$ constant $c$ and where the implicit constant in the second term is uniform in $n$ and $t$.

In order to obtain the actual limiting distribution we prove

THEOREM 2.11. For all $\lambda>0$,

$$
\lim _{t \rightarrow \infty} \frac{t \ln t \tilde{n}}{4} F_{t}^{c}(\lambda)=e^{-\lambda}
$$


where $F_{t}^{c}(\lambda)=P\left(8 \pi N_{A}(t) / m(A) \tilde{n} \ln t>\lambda\right)$.

Proof. By the previous corollary,

$$
\begin{aligned}
\sum_{n=0}^{\infty} & \frac{E\left(-\left(8 \pi N_{A}(t) \cdot s\right) / m(A) \tilde{n} \ln t\right)^{n}}{n !} \\
& =1+\frac{4}{\tilde{n} t \ln t} \sum_{n=1}^{\infty}(-s)^{n}+O\left[\frac{1}{t(\ln t)^{2}} \sum_{n=1}^{\infty}\left(-c^{\prime} s\right)^{n}\right]
\end{aligned}
$$

for $|s|<\rho_{1}<1 / c^{\prime}$ where $|s|<1$. Letting $F_{t}(x)=1-F_{t}^{c}(x)$ we get

$$
\begin{aligned}
\int_{0}^{\infty} e^{-s x} d F_{t}(x)=1-\frac{4}{\tilde{n} t \ln t} \cdot \frac{s}{1+s}+O\left(\frac{1}{t(\ln t)^{2}} \frac{c^{\prime}|s|}{\left(1-c^{\prime}|s|\right.}\right) \\
|s|<\rho_{1}<1 / c^{\prime}
\end{aligned}
$$

or

$\int_{0}^{\infty} \frac{\tilde{n} t \ln t}{4} e^{-s x} F_{t}^{c}(x) d x=\frac{1}{1+s}+O\left(\frac{1}{\ln t\left(1-c^{\prime}|s|\right)}\right), \quad|s|<\rho_{1}<1 / c^{\prime}$.

Thus,

$$
\lim _{t \rightarrow \infty} \int_{0}^{\infty} e^{-s x} \frac{t \ln t \cdot \tilde{n}}{4} F_{t}^{c}(x) d x=\frac{1}{1+s}, \quad|s|<\rho_{1} \leqslant 1 / c^{\prime} .
$$

Now, for all $t$ and for all $s$ in the right half-plane,

$$
\int_{0}^{\infty} e^{-s x} \frac{t \ln t \cdot \tilde{n}}{4} F_{t}^{c}(x) d x
$$

will be bounded and analytic, thereby forming a normal sequence which implies by Vitali's Theorem (cf. Hille [7, Theorem 15.3.1])

$$
\int_{0}^{\infty} e^{-s x} \frac{t \ln t \cdot \tilde{n}}{4} F_{t}^{c}(x) d x \stackrel{\text { u.c.c. }}{\rightarrow} \frac{1}{1+s}=\int_{0}^{\infty} e^{-s x} e^{-x} d x
$$

for all $s$ in the right half-plane which, therefore, implies (cf. Breiman [2, p. 183])

$$
\frac{t \ln t \cdot \tilde{n}}{4} F_{t}^{c}(x) d x \stackrel{w}{\rightarrow} e^{-x} d x \text { for all } x .
$$

Thus, letting $\phi_{t}(x)=(\tilde{n} \ln t / 4) F_{t}^{c}(x)$ we have

$$
\int_{a}^{a+h} \phi_{t}(x) \rightarrow \int_{a}^{a+h} e^{-x} d x=e^{-a}\left(1-e^{-h}\right)
$$

but, now, since $\phi_{t}(x)$ is monotone decreasing, we obtain

$$
\frac{e^{-a}\left(1-e^{-h}\right)}{h}<\liminf _{t \rightarrow \infty} \phi_{t}(a) \leqslant \lim \sup _{t \rightarrow \infty} \phi_{t}(a)<\frac{e^{-a}\left(e^{h}-1\right)}{h}
$$


for positive $a$ and $h$. Since $h$ can be made arbitrarily small we get $\lim _{t \rightarrow \infty} \phi_{t}(a)$ $=e^{-a}$ completing the proof.

It is to be noted that we actually have not been able to prove this result for $\lambda=0$ which would have shown conclusively that $t \ln t$ is the proper normalization for branching Brownian motion in the plane. Remark 4.4 in fact shows our results can not be used to prove the theorem when $\lambda=0$. However, our results do indicate a kind of clustering behavior which is significant.

3. Diffusion on the line. In this chapter we shall consider branching Brownian motion where the state space is now the line. Our basic method of approach is the same as the previous case where the state space was the plane, i.e., first derive an upper estimate for the $n$th moment of the random variable $N_{A}(t)$. However, in order to actually obtain the limiting distribution we must use a different method than that of the previous chapter. Thus, $D=$ $(-\infty, \infty)$ throughout this chapter and defining $\bar{E}_{a}\left[\left(N_{A}(t)\right)^{n}\right]$ as in Definition 2.1 we shall first get an upper bound for $\bar{E}_{a}\left[\left(N_{A}(t)\right)^{n}\right]$. Thus

LEMMA 3.1.

$$
\begin{aligned}
\bar{E}_{a}\left[\left(N_{A}(t)\right)^{n}\right] \leqslant & \frac{n !(m(A))^{n}(\tilde{n})^{n-1}}{(2 \pi)^{n / 2}} t^{n / 2-1} \\
& \cdot\left[1+\frac{(12 r(A)+t)^{n-1}}{\left(t^{+1}\right)^{1 / 2}}\right] \exp \left[-\frac{\left(a-c_{a}\right)^{2}}{2 t}\right]
\end{aligned}
$$

for $n \geqslant 1$, where $\left(a-c_{a}\right)^{2}=\min _{b \in A}(a-b)^{2}$, and $t^{+1}$ denotes $t \vee 1$.

PROOF. Since we are considering one dimension the density

$$
p(t, a, b)=\frac{1}{\sqrt{2 \pi t}} \exp \left[-\frac{(a-b)^{2}}{2 t}\right] .
$$

Thus, for $n=1$,

$$
\bar{E}_{a}\left(N_{A}(t)\right)=\int_{A} p(t, a, b) d b \leqslant \frac{m(A)}{\sqrt{2 \pi t}} \exp \left[\frac{\left(a-c_{a}\right)^{2}}{2 t}\right]
$$

verifying the lemma for $n=1$.

For $n=2$, by definition,

$$
\begin{aligned}
\bar{E}_{a}\left[\left(N_{A}(t)\right)^{2}\right] & =\tilde{n} \int_{A} \int_{A} \int_{0}^{t} \int_{D} p(t-s, a, b) p(s, b, x) p(s, b, y) d b d s d x d y \\
& =\tilde{n} \int_{A} \int_{A} \int_{0}^{t} \frac{1}{\sqrt{2 \pi(t-s)}}
\end{aligned}
$$




$$
\begin{array}{r}
\cdot \frac{1}{2 \pi s}\left[\int_{D} \exp \left[-\frac{(a-b)^{2}}{2(t-s)}\right] \cdot \exp \left[-\frac{(b-x)^{2}}{2 s}\right]\right. \\
\left.\cdot \exp \left[-\frac{(b-y)^{2}}{2 s}\right] d b\right] d s d x d y \\
=\frac{\tilde{n}}{2 \pi} \int_{A} \int_{A} \int_{0}^{t} \frac{1}{\sqrt{s(2 t-s)}} \exp \left[-\frac{(x-y)^{2}}{4 s}\right] \\
\cdot \exp \left[-\frac{(a-(x+y) / 2)^{2}}{2 t-s}\right] d s d x d y
\end{array}
$$

by completing a square. By the definition of $c_{a}$,

$$
\begin{gathered}
\frac{\tilde{n}}{2 \pi} \int_{A} \int_{A} \int_{0}^{t} \frac{1}{\sqrt{s(2 t-s)}} \exp \left[-\frac{(x-y)^{2}}{4 s}\right] \\
\cdot \exp \left[-\frac{\left(a-(x+y)^{2} / 2\right)}{2 t-s}\right] d s d x d y \\
=\frac{\tilde{n}}{2 \pi} \exp \left[-\frac{\left(a-c_{a}\right)^{2}}{2 t}\right] \int_{A} \int_{A} \int_{0}^{t} \frac{1}{\sqrt{s(2 t-s)}} d s d x d y \\
=\frac{\tilde{n}(m(A))^{2}}{4} \exp \left[-\frac{\left(a-c_{a}\right)^{2}}{2 t}\right]
\end{gathered}
$$

verifying the lemma for $n=2$.

Now, assume the lemma for $k<n$, then by Definition 2.1 and the induction hypothesis,

$$
\begin{aligned}
& E_{a}\left[\left(N_{A}(t)\right)^{n}\right] \leqslant \frac{n !(\tilde{n})^{n-1}(m(A))^{n}}{(2 \pi)^{n / 2} \cdot 2} \\
& \cdot \sum_{k=1}^{n-1} \int_{0}^{t \cdot} \frac{s^{n / 2-2}}{\sqrt{2 \pi(t-s)}}\left[1+\frac{R(k)}{\left(s^{+1}\right)^{1 / 2}}\right]\left[1+\frac{R(n-k)}{\left(s^{+1}\right)^{1 / 2}}\right] \\
& \cdot\left\{\int_{-\infty}^{\infty} \exp \left[-\frac{(a-b)^{2}}{2(t-s)}\right] \cdot \exp \left[-\frac{\left(b-c_{b}\right)^{2}}{s}\right] d b\right\} d s
\end{aligned}
$$

where $R(k)=\left[12(r(A))^{2}+5\right]^{k-1}$. Completing a square and changing to 
polar coordinates implies

$$
\begin{aligned}
& \int_{-\infty}^{\infty} \exp \left[-\frac{(a-b)^{2}}{2(t-s)}\right] \cdot \exp \left[-\frac{\left(b-c_{b}\right)^{2}}{s}\right] d b \\
& <2 \cdot \exp \left[-\frac{\left(a-c_{a}\right)^{2}}{2 t}\right]\left\{\int_{0}^{2 r(A)(t-s) /(2 t-s)} d b+\int_{2 r(A)(t-s) /(2 t-s)}^{\infty}\right. \\
& \left.\cdot \exp \left[-\left(b-\frac{2 \hat{c}_{b}(t-s)}{2 t-s}\right)^{2}\left(\frac{2 t-s}{2 s(t-s)}\right)\right] d b\right\} \\
& =\exp \left[-\frac{\left(a-c_{a}\right)^{2}}{2 t}\right]\left\{\frac{4 r(A)(t-s)}{2 t-s}+\sqrt{\frac{2 \pi s(t-s)}{2 t-s}}\right\} .
\end{aligned}
$$

Thus

$$
\begin{aligned}
\bar{E}_{a}\left[\left(N_{A}(t)\right)^{n}\right]< & \sum_{k=1}^{n-1} c(n) \int_{0}^{t} \frac{s^{n / 2-2}}{\sqrt{2 \pi(t-s)}}\left(1+\frac{R(k)}{\sqrt{s^{+1}}}\right) \\
& \cdot\left(1+\frac{R(n-k)}{\sqrt{s^{+1}}}\right)\left(\frac{2 r(A)(t-s)}{2 t-s}+\frac{1}{2} \sqrt{\frac{2 \pi s(t-s)}{2 t-s}}\right) d s
\end{aligned}
$$

where

$$
c(n)=\frac{n !(\tilde{n})^{n-1}(m(A))^{n}}{(2 \pi)^{n / 2}} \exp \left[-\frac{\left(a-c_{a}\right)^{2}}{2 t}\right] .
$$

Since $n \geqslant 3$ we get

$$
\begin{aligned}
\bar{E}_{a}\left[\left(N_{A}(t)\right)^{n}\right]< & \frac{n !(\tilde{n})^{n-1}(m(A))^{n}}{(2 \pi)^{n / 2}} \\
& \cdot\left[\exp \left[-\frac{\left(a-c_{a}\right)^{2}}{2 t}\right] t^{n / 2-1}\left[1+\frac{(12 r(A)+5)^{n-1}}{\left(t^{+1}\right)^{1 / 2}}\right]\right.
\end{aligned}
$$

completing the proof.

REMARK 3.2. By the same method we can replace the $n$ ! by $l(n)$ where $l(n)=\frac{{ }_{1} F_{1}(1 / 2 ;(n+1) / 2, z)}{n-1} \sum_{k=1}^{n-1}\left(\begin{array}{l}n \\ k\end{array}\right) l(k) l(n-k), \quad l(0)=0, \quad l(1)=1$ at $z=-1$, where ${ }_{1} F_{1}$ is the confluent hypergeometric series, since 
BRANCHING RANDOM FIELDS

379

$$
\int_{0}^{t} \frac{s^{(n-3) / 2}}{\sqrt{2 t-s}} d s=\frac{2 t^{n / 2-1}}{n-1}{ }_{1} F_{1}\left(\frac{1}{2} ; \frac{n+1}{2},-1\right) .
$$

In order to derive an asymptotic estimate for $\bar{E}_{a}\left[\left(N_{A}(t)\right)^{n}\right]$ we wish to find its density. Therefore

Definition 3.3. Let

$$
\begin{aligned}
& Q_{n}\left(a, t, x_{1}, x_{2}, \ldots, x_{n}\right)=\frac{\tilde{n}}{2} \sum_{\sigma \in \pi_{n}} \sum_{k=1}^{n-1} \frac{1}{k !} \cdot \frac{1}{(n-k) !} \\
& \quad \cdot \int_{0}^{t} \int_{D} p(t-s, a, b) Q_{k}\left(b, s, x_{\sigma_{1}}, \ldots, x_{\sigma_{k}}\right) Q_{n-k}\left(b, s, x_{\sigma_{k+1}}, \ldots, x_{\sigma_{n}}\right) d b d s,
\end{aligned}
$$

for $n \geqslant 2$, and $Q_{1}(a, t, k)=p(t, a, k)$ where $\pi_{n}$ is the permutation group on $n$ letters (and where $\sigma_{i}$ is the image of $i$ under the permutation $\sigma$ ).

LEMMA 3.4.

$$
\bar{E}_{a}\left[\left(N_{A}(t)\right)^{n}\right]=\int_{A} \cdots \int_{A} Q_{n}\left(a, t, x_{1}, \ldots, x_{n}\right) d x_{1} \cdots d x_{n} .
$$

For $n=1$, it is clear since $\bar{E}_{a}\left[\left(N_{A}(t)\right)\right]=\int_{A} p(t, a, b) d b$.

Assume the lemma for $k<n$, then using Definition 2.1

$$
\begin{aligned}
\bar{E}_{a}\left[\left(N_{A}(t)\right)^{n}\right]= & \frac{\tilde{n}}{2} \sum_{k=1}^{n-1}\left(\begin{array}{l}
n \\
k
\end{array}\right) \int_{0}^{t} \int_{D} p(t-s, a, b) \\
& \cdot \int_{A^{k}} Q_{k}\left(b, s, x_{1}, \ldots, x_{k}\right) d x_{1} \cdots d x_{k} \\
& \cdot \int_{A^{n-k}} Q_{n-k}\left(b, s, x_{k+1}, \ldots, x_{n}\right) d x_{k+1} \cdots d x_{n} \\
= & \int_{A^{n}} \frac{\tilde{n}}{2} \sum_{\sigma \in \pi_{n}} \sum_{k=1}^{n-1} \frac{1}{k !} \frac{1}{(n-k) !} \int_{0}^{t} \\
& \cdot \int_{D} p(t-s, a, b) Q_{k}\left(b, s, x_{\sigma_{1}}, \ldots, x_{\sigma_{k}}\right) \\
& \cdot Q_{n-k}\left(b, s, x_{\sigma_{k+1}}, \ldots, x_{\sigma_{n}}\right) d b d s d x_{\sigma_{1}} \cdots d x_{\sigma_{n}} \\
= & \int_{A^{n}} Q_{n}\left(a, t, x_{1}, \ldots, x_{n}\right) d x_{1} \cdots d x_{n} .
\end{aligned}
$$

LEMMA 3.5 .

$$
Q_{n}\left(a, t, x_{1}, \ldots, x_{n}\right)=Q_{n}\left(\frac{a}{\sqrt{t}}, 1, \frac{x_{1}}{\sqrt{t}}, \ldots, \frac{x_{n}}{\sqrt{t}}\right) \cdot t^{n / 2-1}
$$

Proof. For $n=1$, 


$$
Q_{1}\left(\frac{a}{\sqrt{t}}, 1, \frac{x}{\sqrt{t}}\right)=\frac{1}{\sqrt{2 \pi}} \exp \left[\frac{-(a / \sqrt{t}-x / \sqrt{t})^{2}}{2}\right]=\sqrt{t} p(t, a, x) \text {. }
$$

For $n>1$, by a change of variables and Definition 3.3,

$$
\begin{aligned}
Q_{n}\left(a, t, x_{1}, \ldots, x_{n}\right)=\frac{\tilde{n}}{2} \sum_{\sigma \in \pi_{n}} \sum_{k=1}^{n-1} \frac{1}{k !} \cdot \frac{1}{(n-k) !} t^{3 / 2} \\
\cdot \int_{0}^{1} \int_{D} p(t-s t, a, b \sqrt{t}) Q_{k}\left(b \sqrt{t}, s t, x_{\sigma_{1}}, \ldots, x_{\sigma_{k}}\right) \\
\cdot Q_{n-k}\left(b \sqrt{t}, s t, x_{\sigma_{k}}, \ldots, x_{\sigma_{n}}\right) d b d s .
\end{aligned}
$$

Assuming the lemma for $k<n$ implies likewise that

$$
Q_{k}\left(a, s t, x_{1}, \ldots, x_{k}\right)=Q_{k}\left(\frac{a}{\sqrt{t}}, s, \frac{x_{1}}{\sqrt{t}}, \ldots, \frac{x_{k}}{\sqrt{t}}\right) t^{k / 2-1} .
$$

Since $p(t-s t, a, b \sqrt{t})=p(1-s, a / \sqrt{t}, b) \cdot t^{-1 / 2}$ the lemma follows.

LEMMA 3.6. $Q_{n}\left(a, t, x_{1}, \ldots, x_{n}\right)<c_{n}$ for $0 \leqslant t<1$ and $n>1$.

Proof. For $n=2$,

$$
\begin{aligned}
& Q_{2}\left(a, t, x_{1}, x_{2}\right) \\
& \quad=\tilde{n} \int_{A^{2}} \int_{0}^{t} \int_{D} p(t-s, a, b) p(s, b, x) p(s, b, y) d b d s d x d y<c<c_{2} .
\end{aligned}
$$

as in Lemma 3.1. Assume the lemma for $k<n$, then, since

$$
\begin{aligned}
& Q_{n}\left(a, t, x_{1}, \ldots, x_{n}\right)=\frac{\tilde{n}}{2} \sum_{\sigma \in \pi_{n}} \sum_{k=1}^{n-1} \frac{1}{k !} \\
& \cdot \frac{1}{(n-k) !} \int_{0}^{t} \int_{D} p(t-s, a, b) Q_{k}\left(b, s, x_{\sigma_{1}}, \ldots, x_{\sigma_{k}}\right) \\
& \cdot Q_{n-k}\left(b, s, x_{\sigma_{k+1}}, \ldots, x_{\sigma_{n}}\right) d b d s
\end{aligned}
$$

if $k \neq 1, n-1$ the lemma follows. In the case $k=1$ or $n-1$ the integral is bounded by

$$
c_{n-1} \int_{0}^{t} \int_{D} p(t-s, a, b) p\left(s, b, x_{\sigma_{1}}\right) d b d s \leqslant c_{n-1}
$$

LEMMA 3.7. $\forall n, Q_{n}\left(a, 1, x_{1}, \ldots, x_{n}\right) \in C\left(R^{n+1}\right)$.

ProOF. The lemma follows from the previous lemma and an induction.

We have, therefore, shown 
THEOREM 3.8.

$$
\begin{aligned}
\bar{E}_{a}\left[\left(N_{A}(t)\right)^{n}\right] & =\int_{A^{n}} Q_{n}\left(a, t, x_{1}, \ldots, x_{n}\right) d x_{1} \cdots d x_{n} \\
& =t^{n / 2-1} \int_{A^{n}} Q_{n}\left(\frac{a}{\sqrt{t}}, 1, \frac{x_{1}}{\sqrt{t}}, \ldots, \frac{x_{n}}{\sqrt{t}}\right) d x_{1} \cdots d x_{n} \\
& \sim t^{n / 2-1}(m(A))^{n} l(n)
\end{aligned}
$$

(for all $n$ as $t \rightarrow \infty)$ where $l(n)=Q_{n}(0,1,0, \ldots, 0)$.

REMARK 3.9. By methods similar to those used in proving Lemma 3.1 one can show that there exists a universal constant $\alpha$ satisfying $l(n)>\alpha^{n}>0$.

LEMMA 3.10.

$$
\begin{aligned}
\bar{E}_{a}\left[\left(N_{A}(t)\right)^{n}\right] \leqslant & E_{a}\left[\left(N_{A}(t)\right)^{n}\right] \\
< & \bar{E}_{a}\left[\left(N_{A}(t)\right)^{n}\right]+q(n)[m(A) \vee 1]^{n} \\
& \cdot\left[t^{(n-3) / 2} \exp \left[-\frac{\left(a-c_{a}\right)^{2}}{2 t}\right] \cdot \chi_{(t>1)}+\chi_{(t<1)}\right]
\end{aligned}
$$

where $q(n)$ is $O\left(c^{n-1} \cdot n !\right)$ for some $c>0$.

Proof. The method is the same as that used in proving Lemma 2.9. Use Lemma 2.7 and the following estimate which follows from Lemma 3.1:

$$
\begin{aligned}
& \bar{E}_{a}\left[\left(N_{A}(t)\right)^{n}\right] \leqslant \frac{(m(A))^{n}(\tilde{n})^{n-1} n !}{(2 \pi)^{(n-1) / 2}}(12 r(A)+6)^{n-1} \chi_{(t<1)} \\
& +\frac{(m(A))^{n}(\tilde{n})^{n-1} \cdot n !}{(2 \pi)^{n / 2}}(12 r(A)+6)^{n-1} \exp \left[-\frac{\left(a-c_{a}\right)^{2}}{2 t}\right] \cdot t^{n / 2-1} \cdot \chi_{(t>1)}
\end{aligned}
$$

for $n>0$.

Now, if we set

since we have shown that

$$
\bar{F}_{t}^{c}(\lambda)=P\left(\frac{N_{A}(t)}{m(A) \sqrt{t}}>\lambda\right)
$$

$$
\sum_{n=0}^{\infty} \frac{E_{a}\left[\left[-\frac{\sqrt{2 \pi} N_{A}(t)}{m(A) \tilde{n} \sqrt{t}} s\right]^{n}\right]}{n !}<1+\frac{1}{\tilde{n} t}\left[-\frac{s}{(1+s)}\right]+O\left(\frac{1}{t^{3 / 2}}\right)
$$


$\tilde{n} t \int_{0}^{\infty} e^{-s x} \bar{F}_{t}^{c}(x) d x$ will be bounded and analytic, thereby forming a normal sequence for $s$ in the right half plane. However, we have shown in Theorem 3.8 that all its derivatives converge at $s=0$ which, therefore, shows that $t \int_{0}^{\infty} e^{-s x} \bar{F}_{t}^{c}(x) d x$ will converge weakly for $s$ in the right half-plane. Thus, using the same arguments as in Theorem 2.11 we have shown,

THEOREM 3.11. $\lim _{t \rightarrow \infty} t \bar{F}_{t}^{c}(\lambda)=F(\lambda)$ on $\{\lambda: F(\lambda)$ is continuous $\}$ where $F(\lambda)$ is a decreasing function on $(0, \infty)$ satisfying

$$
\int_{0}^{\infty} y^{n}(-F(d y))=l(n)
$$

for $n \geqslant 1$.

REMARK 3.12. In order to determine a distribution we have calculated some of the lower order moments and have found

$$
\begin{aligned}
E\left(N_{A}(t)\right) & =\frac{m(A)}{\sqrt{2 \pi t}}+O\left(\frac{1}{t}\right), \\
E\left[\left(N_{A}(t)\right)^{2}\right] & =\frac{\tilde{n}(m(A))^{2}}{4}+O\left(\frac{1}{\sqrt{t}}\right), \\
E\left[\left(N_{A}(t)\right)^{3}\right] & =\left(\frac{\pi}{5}+\sqrt{3}-1\right)(\tilde{n})^{2}(m(A))^{3} t^{1 / 2}+O(1) .
\end{aligned}
$$

From this it follows that

$$
\begin{gathered}
-\int_{0}^{\infty} \lambda d F(\lambda)=1, \quad-\int_{0}^{\infty} \lambda^{2} d F(\lambda)=\frac{\pi}{2}, \\
-\int_{0}^{\infty} \lambda^{3} d F(\lambda)=(2 \pi)^{3 / 2}\left[\frac{\pi}{6}+\sqrt{3}-1\right] .
\end{gathered}
$$

Thus $F(\lambda)$ is not an exponential, Mittag-Lefler or gamma distribution. It is also interesting to note that the rate of decay appears to be at a rate of $1 / t$ which is similar to the stationary branching process, unlike the case for diffusion in the plane.

4. An initial random field. In this chapter we shall consider a branching random field. The individual particles will still move about like Brownian motion. However, rather than assume a single initial particle we will assume that originally a discrete but random number of particles are introduced and that given the initial state, the particles then proceed to move and branch independently of each other. Since we start with more than one particle rather than use moment generating functions we use cumulant generating functions and it turns out that due to the initial state we get a different rate of decay than for the case of a single initial particle.

To fix the process, we associate with the process the Borel measure 
$\mu(A)=E\left(N_{A}(0)\right)$ corresponding with the random variable $N_{A}(0)$. Using step function approximations one obtains $E\left(N_{A}(t)\right)=\int_{D} E_{a}\left(N_{A}(t)\right) d \mu(a)$. In the special case where one assumes that for disjoint sets $A_{1}, \ldots, A_{n}$ the random variables $\left\{N_{A_{1}}(0), N_{A_{2}}(0), \ldots, N_{A_{n}}(0)\right\}$ are mutually independent, initially no two particles are located at the same point, and $\mu(A)$ is continuous then (cf. Karlin [9, p. 338]) each $N_{A_{i}}(0)$ has a Poisson distribution. However in our case we do not require an initial Poisson field; we only require a field where $\mu(A)$ is Lebesgue measure.

Denote by $X_{i}(t)$ the random variable which gives the number of particles in $A$ at time $t$ which are descendants of an initial particle at state $\xi_{i}$ for $i=1, \ldots, m$ and $X(t)$ the number in $A$ for the given initial position $\xi=\left(\xi_{1}, \ldots, \xi_{m}\right)$. Then by independence, $E\left(e^{s X}\right)=E\left(e^{s X_{1}}\right) \cdots E\left(e^{s X_{m}}\right)$ or $\log f_{\xi}(s)=\sum_{i=1}^{m} \log f_{\xi}(s)$ where $f_{\xi}(x)=E\left(e^{s X_{i}}\right)$ and $f_{\xi}(s)=E\left(e^{s X}\right)$.

In our case, if $\xi=\left(\xi_{1}, \xi_{2}, \ldots, \ldots\right)$ denotes the initial state of the system, which is in general infinite, then

$$
\begin{aligned}
\log f(s) & =E\left[\log f_{\left(\xi_{1}, \xi_{2}, \ldots, \ldots\right)}(s)\right]=E\left[\sum_{i=1}^{\infty} \log f_{\xi}(s)\right] \\
& =E\left[\int_{D} \log f_{a}(s) N_{d a}(0)\right]=\int_{D} \log f_{a}(s) \mu(d a)
\end{aligned}
$$

since in general $E\left[\sum_{i=1}^{\infty} f\left(\xi_{i}\right)\right]=\int_{D} f(a) \mu(d a)$ when $\int_{D}|f(a)| \mu(d a)<\infty$ and

$$
\begin{aligned}
\int\left|\log E_{a} e^{s N_{1}(t)}\right| d a & =\int\left|\log \left[1+E_{a}\left(e^{s N_{1}(t)}-1\right)\right]\right| d a \\
& <\int E_{a}\left(e^{s N_{1}(t)}-1\right) d a<\infty
\end{aligned}
$$

by Lemmas 3.1, 3.10 for $s>0, t>4$.

In general, if $f(s)=E\left(e^{s X}\right)$ for a random variable $X$ for which $f( \pm \varepsilon)<\infty$ for some $\varepsilon>0$ then $f(s)=\sum_{n=0}^{\infty}\left(\alpha_{n} s^{n} / n !\right)$ where $\alpha_{n}=E\left(X^{n}\right)$ is the $n$th moment and $\log f(s)=\Sigma_{n>1}\left(\chi_{n} s^{n} / n\right.$ !) where $\chi_{n}$ is the $n$th cumulant of $X$. The relation between $\chi_{n}$ and $\alpha_{n}$ is given by (cf. Lukacs [10, p. 34])

$$
\chi_{n}=\sum(-1)^{k-1} \frac{(k-1) ! n ! \alpha_{k_{1}}^{i_{1}} \cdots \cdots \alpha_{k_{s}}^{i_{s}}}{i_{1} !\left(k_{1} !\right)^{i_{1}} \cdots i_{s} !\left(k_{s} !\right)^{i_{s}}}
$$

where the summation is over all partitions of $n$ where $i_{1}+\cdots+i_{s}=k$ and $i_{1} k_{1}+i_{2} k_{2}+i_{s} k_{s}=n$. Thus,

THEOREM 4.1. In case we start out with a random field of particles diffusing independently in $D$, the plane, then

$$
\lim _{t \rightarrow \infty} \frac{\tilde{n} \cdot \log t}{8 \pi} G_{t}^{c}(\lambda)=e^{-\lambda}, \quad \forall \lambda>0
$$


where

$$
G_{i}^{c}(\lambda)=P\left(\frac{N_{A}(t) \cdot 8 \pi}{\tilde{n} m(A) \log t}>\lambda\right)
$$

Proof. By Corollary 2.10,

$$
\begin{aligned}
\alpha_{n}(a)= & E\left(\left(N_{A}(t)\right)^{n} \mid X_{0}=a\right) \\
= & \frac{n !(\log t)^{n-1}(m(A))^{n}(\tilde{n})^{n-1}}{(2 \pi)^{n} 4^{n-1} t} \cdot \exp \left[-\frac{\left(a-c_{a}\right)^{2}}{2 t}\right] \\
& +O\left(\frac{c^{n} \cdot n !}{t} \cdot(\log t)^{n-2}\right) \exp \left[-\frac{\left(a-c_{a}^{\prime}\right)^{2}}{2 t}\right]
\end{aligned}
$$

Therefore, if we have a product $\alpha_{i}(a) \cdot \alpha_{j}(a)$ we will get a $t^{2}$ in the denominator. Also it is clear (cf. Gnedenko and Kolmogorov [6, p. 65]) that the sum of the coefficients of all terms in $\chi_{n}(a)$ besides $\alpha_{n}(a)$ is $<3^{n}$ where $\chi_{n}(a)$ is the corresponding $n$th cumulant. Thus,

$$
\begin{aligned}
\chi_{n}(a)= & \frac{n !(\log t)^{n-1}(m(A))^{n}(\tilde{n})^{n-1}}{(2 \pi)^{n} 4^{n-1} t} \exp \left[-\frac{\left(a-c_{a}\right)^{2}}{2 t}\right] \\
& +O\left(\frac{c_{2}^{n} \cdot n !(\log t)^{n-2}}{t}\right) \exp \left[-\frac{\left(a-c_{a}^{\prime}\right)^{2}}{2 t}\right]
\end{aligned}
$$

However, the $n$th cumulant of $N_{A}(t)$ given an initial random field is given by $\chi_{n}=\int_{D} \chi_{n}(a) d a$. Hence,

$$
\begin{aligned}
\log E\left(e^{s N_{1}(t)}\right) & =\sum_{n=1}^{\infty} \frac{\chi_{n}}{n !} s^{n}=\sum_{n=1}^{\infty} \frac{s^{n}}{n !} \int_{D} \chi_{n}(a) d a \\
= & \frac{8 \pi}{\tilde{n} \log t} \sum_{n=1}^{\infty}\left(\frac{\tilde{n} m(A) \cdot \log t s}{8 \pi}\right)^{n}+O\left(\frac{1}{(\log t)^{2}} \sum_{n=1}^{\infty}(\operatorname{cs} \log t)^{n}\right)
\end{aligned}
$$

Thus,

$$
\log E\left(\exp \left[\frac{8 \pi s}{\tilde{n} m(A) \log t} N_{A}(t)\right]\right)=\frac{8 \pi \cdot s}{\tilde{n} \log t(1-s)}+O\left(\frac{c s}{(1-s)(\log t)^{2}}\right) .
$$

Therefore,

$$
\begin{aligned}
f\left(\frac{8 \pi s}{\tilde{n} m(A) \log t}\right) & =\exp \left[\frac{8 \pi s}{\tilde{n} \log t(1-s)}+\frac{c s}{(\log t)^{2}(1-s)}\right] \\
& =1+\frac{8 \pi s}{\tilde{n} \log t(1-s)}+O\left(\frac{1}{(\log t)^{2}}\right) .
\end{aligned}
$$


Now, proceeding as in the proof of Theorem 2.8, we obtain the theorem.

In case we start with an initial random field diffusing independently in $D=(-\infty, \infty)$ we obtain,

THEOREM 4.2.

$$
\lim _{t \rightarrow \infty} \frac{\bar{c} \sqrt{t} \tilde{n}}{\sqrt{2 \pi}} \bar{G}_{t}^{c}(\lambda)=\tilde{F}(\lambda)
$$

on $\{\lambda: \tilde{F}(\lambda)$ is continuous $\}$ for some distribution $\tilde{F}(\lambda)$ and where

$$
\bar{G}_{l}^{c}(\lambda)=P\left(\frac{N_{A}(t) \cdot \sqrt{2 \pi}}{m(A) \tilde{n} \sqrt{t}}>\lambda\right) \text { for some constant } \bar{c} .
$$

REMARK 4.3. As before we calculated and found for this case

$$
\begin{gathered}
E\left(N_{A}(t)\right)=m(A)+O\left(\frac{1}{\sqrt{t}}\right), \\
E\left[\left(N_{A}(t)\right)^{2}\right]=\frac{\tilde{n}(m(A))^{2} t^{1 / 2}}{\pi^{1 / 2}}+O(1), \\
E\left[\left(N_{A}(t)\right)^{3}\right]=\frac{\tilde{n} t(m(A))^{3}}{2}+O(\sqrt{t}), \\
E\left[\left(N_{A}(t)\right)^{4}\right]=3(\tilde{n})^{3} t^{3 / 2}(m(A))^{4} \\
\cdot\left\{\frac{16}{3} \tan ^{-1}(\sqrt{2})+\frac{4 \sqrt{3}}{3}-\frac{4 \sqrt{2}}{3}-\frac{16}{9} \pi\right. \\
\left.-\frac{1}{2} \ln \left(\frac{\sqrt{2}-1}{\sqrt{2}+1}\right)+\left(\frac{4}{3}+\frac{3 \sqrt{2}}{3}\right) \sin ^{-1}\left(\frac{\sqrt{3}}{3}\right)\right\}+O(t),
\end{gathered}
$$

and thus once again have obtained some exotic distribution where $\tilde{F}(\lambda)$ is not the same as the distribution $F(\lambda)$ obtained in the case of a single initial particle.

ReMARK 4.4. Lastly we show that the limit theorems we have obtained (2.11, 3.11, 4.1, 4.2) cannot be shown for $\lambda=0$ given just the bounds on the moments which we have obtained. We give a counterexample for Theorem 2.11 in case $\lambda=0$. Consider $X_{t} \cong C \log t$ on a set of probability $1 / t \log t$ and $X_{t} \cong C e^{-t}$ on a set of positive probability, then

$$
P\left(\frac{X_{t}}{\log t}>\varepsilon\right) \rightarrow \frac{c}{t \log t} P\left(\frac{X_{t}}{\log t}>0\right)=1 .
$$


Appendix 1. In this appendix we furnish a proof of Lemma 1.1:

LEMMA 1.1.

$$
\begin{aligned}
& E_{a}\left[\left(N_{A}(t)\right)^{k}\right]=\int_{A} m(t, a, b) d b \\
& +\sum_{r=2}^{k} \frac{\tilde{n}_{r}}{r !} \sum_{\left(h_{1}, \ldots, h_{r}\right)} \frac{k !}{h_{1} ! h_{2} ! \cdots h_{r} !} \int_{0}^{t} \int_{D} m(t-s, a, b) \\
& \sum_{j=1}^{r} h_{j}=k \\
& 1<h_{j}<k \\
& \cdot E_{b}\left[\left(N_{A}(s)\right)^{h_{1}}\right] \cdots E_{b}\left[\left(N_{A}(s)\right)^{h_{r}}\right] d b d s
\end{aligned}
$$

Proof. Let $u_{k}(a, t)=E_{a}\left[\exp \left(-v_{1} \hat{f}_{1}\left(x_{t}\right)-\cdots-v_{k} \hat{f}_{k}\left(x_{t}\right)\right]\right.$ where $f_{i} \geqslant 0$ and $f_{i} \in B_{0}(D)$. The branching property is $u_{k}(a, t)=\prod_{i=1}^{n} u_{k}\left(a_{i}, t\right)$. Now, by the strong Markov property, since $E(E(X \mid A) \mid B)=E(X \mid B)$ for $B \subset A, B$ and $A \sigma$-algebras,

$$
\begin{array}{r}
u_{k}(a, t)=E_{a}\left(\exp \left[-v_{1} \hat{f}_{1}\left(x_{t}\right)-\cdots-v_{k} \hat{f}_{k}\left(x_{t}\right)\right] \cdot \chi_{(\beta>t)}\right) \\
+E_{a}\left(\chi _ { ( \beta < t ) } \cdot \sum _ { n = 0 } ^ { \infty } \int _ { D ^ { n } } \prod _ { i = 1 } ^ { n } E \left(\operatorname { e x p } \left[-v_{1} \hat{f}_{1}\left(x_{t}\right)\right.\right.\right. \\
\left.\left.\left.-\cdots-v_{k} \hat{f}_{k}\left(x_{t}\right)\right] \mid \chi_{\beta}=a_{i}\right) \pi_{n}\left(x_{\beta}, d y\right)\right)
\end{array}
$$

where the first term represents no branch by time $t$ and the 2 nd a branch into $n$ particles at time $\beta$ located at $y=\left(a_{1}, \ldots, a_{n}\right)$.

Differentiating with respect to $v_{1}, \ldots, v_{k}$ and letting $v_{1}, \ldots, v_{k} \rightarrow 0$ we obtain:

$$
\begin{aligned}
& B_{t}^{(k)}\left(a, f_{1}, \ldots, f_{k}\right)=E_{a}\left(\hat{f}_{1}\left(x_{t}\right) \cdots \hat{f}_{k}\left(x_{t}\right) \cdot \chi_{(\beta>t)}\right) \\
& +E_{a}\left[\chi _ { ( \beta < t ) } \cdot \sum _ { n = 0 } ^ { \infty } \int _ { D ^ { n } } \left\{\sum_{i=1}^{n} E_{a_{i}}\left(\hat{f}_{1}\left(x_{t}\right) \cdots \hat{f}_{k}\left(x_{t}\right)\right)\right.\right. \\
& +\sum_{r=2}^{k} \frac{1}{r !} \\
& \sum_{j=1}^{r} h_{j}=k, h_{j} \geqslant 1 \\
& \cdot \sum_{i_{1} \neq} \cdots \sum_{\neq i_{r}} E_{a_{i_{1}}}\left(\hat{f}_{u_{1}} \cdots \hat{f}_{u_{u_{1}}}\right) \cdots \\
& \left.\left.E_{a_{i r}}\left(\hat{f}_{(}, \cdots \hat{f}_{u_{r}}\right)\right\} \pi_{n}\left(X_{\beta}, d y\right)\right]
\end{aligned}
$$


since we are uninterested in order where the term $f_{u_{1}} \cdots \cdots f_{u_{k}}$ represents some average of permutations of $f_{1} \cdots f_{k}$. Now since lifetimes are exponentially distributed we obtain:

$$
\begin{aligned}
B_{t}^{(k)}\left(a, f_{1}, \ldots, f_{k}\right)= & T_{t}^{0}\left(f_{1}, \ldots, f_{k}\right)(a) \\
+\int_{0}^{t} e^{-v_{s}} E_{a}\left[w B_{t-s}^{(k)}\left(a, f_{1}, \ldots, f_{k}\right)+\sum_{r=2}^{k} \frac{1}{r !} \cdot\right. & \\
& \sum_{\substack{\left(h_{1}, \ldots, h_{r}\right) \\
h_{j}=k, h_{j} \geqslant 1}} \frac{k !}{h_{1} ! \cdots h_{r} !} \tilde{n}_{r}\left(x_{s}, B_{t-s}^{\left(h_{1}\right)}\left(y_{1}, f_{k}, \ldots, f_{k_{h_{1}}}\right),\right. \\
& \left.\left.\ldots, B_{t-s}^{\left(h_{r}\right)}\left(y_{r}, f_{(}, \ldots, f_{k_{r}}\right)\right)\right] d s
\end{aligned}
$$

where $T_{t}^{0}$ is the expectation operator of the unbranched process (which in the case of Brownian motion is $e^{-v t}$ times the Brownian semigroup),

$$
w f(a)=V \sum_{n=0}^{\infty} \int_{D^{n}} \sum_{i=1}^{n} f\left(y_{i}\right) \pi_{n}(a, d y)
$$

and

$$
\tilde{n}_{r}\left(a, f_{1}, \ldots, f_{r}\right)=V \sum_{n=0}^{\infty} \int_{D^{n}} \sum_{i_{1} \neq i_{2} \neq} \sum_{\neq i_{r}} \cdots \sum_{1}() \cdots f_{r}() \pi_{n}(a, d y) .
$$

Next, we take Laplace transforms and obtain

$$
\begin{aligned}
I_{\lambda}\left(a, f_{1}, \ldots, f_{k}\right) & =\int_{0}^{\infty} e^{-\lambda t} B_{t}^{(k)}\left(a, f_{1}, \ldots, f_{k}\right) d t \\
& =+R_{\lambda}^{0} f_{1} \cdots f_{k}(a)+R_{\lambda}^{0} w I_{\lambda}+R_{\lambda}^{0}\left(q_{\lambda}\right)
\end{aligned}
$$

where $R_{\lambda}^{0}$ is the Laplace transform of $T_{t}^{0}$ and $q_{\lambda}$ is the Laplace transform in $t$ of

$$
\begin{aligned}
\sum_{r=2}^{k} \frac{1}{r !} \sum_{\substack{\left(h_{1}, \ldots, h_{r}\right) \\
\sum h_{j}=k, h_{j} \geqslant 1}} \frac{k !}{h_{1} ! \cdots h_{r} !} \tilde{n}_{r}\left(x_{s}, B_{i-s}^{\left(h_{1}\right)}\left(y_{1}, f_{u_{1}}, \ldots, f_{u_{h_{1}}}\right), \ldots,\right. \\
\left.B_{i-s}^{\left(h_{r}\right)}\left(y_{r}, f_{\left.u_{(}\right)}, \ldots, f_{u_{h_{r}}}\right)\right) .
\end{aligned}
$$

Now, by Hille-Yosida, since $A^{0}=A-V I$ (cf. [16]), we obtain

$$
(M-\lambda I) I_{\lambda}\left(a, f_{1}, \ldots, f_{k}\right)=-f_{1} \cdots f_{k}(a)-q_{\lambda}\left(a, f_{1}, \ldots, f_{k}\right)
$$

where $M=A+V(\bar{m}-I)$ as before. By [17, Appendix 1] $Q(\lambda)(M-\lambda I)=$ - I where $Q(\lambda)$ is the Laplace transform of $\int_{D} m(t, a, b) f(b) d b$ and since we are in the critical case,

$$
I_{\lambda}\left(a, f_{1}, \ldots, f_{k}\right)=Q(\lambda) f_{1} \cdots f_{k}(a)+Q(\lambda) q_{\lambda}\left(a, f_{1}, \ldots, f_{k}\right) .
$$


Inverting (1) we get on the left side $B^{(k)}\left(a, f_{1}, \ldots, f_{k}\right)$, the first term on the right side is $\int_{D} m(t, a, b) f_{1} \cdots f_{k}(b) d b$ (cf. [16, Theorem 2.1]) and 2nd term on the right is

$$
\begin{aligned}
& \int_{D} \int_{D}^{t} m(t-s, a, b) \\
& \cdot \sum_{r=2}^{k} \sum_{\substack{\left(h_{1}, \ldots, h_{r}\right) \\
\sum_{j=1} h_{j}=k, h_{j}>1}} \frac{k !}{h_{1} ! \cdots h_{r} !} \frac{\tilde{n}_{r}}{r !}\left[B_{s}^{\left(h_{1}\right)}\left(y_{1}, f_{u_{1}}, \ldots, f_{u_{h_{1}}}\right)\right. \\
& \\
&\left.\cdots B^{\left(h_{1}\right)}\left(y_{r}, f_{(}, \ldots, f_{u_{m}}\right)\right] d s d b .
\end{aligned}
$$

In the special case we are interested in $f_{1}=\cdots=f_{k}=\chi_{1}$. We thus obtain:

$$
\begin{aligned}
E_{a}\left[\left(N_{A}(t)\right)^{k}\right] & =\int_{A} p(t, a, b) d b \\
+\sum_{r=2}^{k} \frac{\tilde{n}_{r}}{r !} & \sum_{\substack{\left(h_{1}, \ldots, h_{r}\right) \\
\sum_{j=1}^{n} h_{j}=k}} \frac{k !}{h_{1} ! \cdots h_{r} !} \\
& \cdot \int_{D} \int_{0}^{t} p(t-s, a, b) E_{b}\left[\left(N_{A}(s)\right)^{h_{1}}\right] \cdots E_{b}\left[\left(N_{A}(s)\right)^{h_{r}}\right] d s d b
\end{aligned}
$$

where $\tilde{n}_{r}$ reduced to $V \sum_{n-r}^{\infty} n(n-1) \cdots(n-r+1) p_{n}$ and we let $\tilde{n}_{2}=\tilde{n}$.

\section{BIBLIOGRAPHY}

1. K. Athreya and P. Ney, Branching processes, Springer-Verlag, Berlin and New York, 1972.

2. L. Breiman, Probability, Addison-Wesley, Reading, Mass., 1968.

3. J. Crow and M. Kimura, An introduction to population genetics theory, Harper and Row, New York, 1970.

4. W. Ewens, Population genetics, Methuen, New York, 1969.

5. J. Felsenstein, A pain in the torus: Some difficulties with models of isolation by distance, Amer. Natur. 109 (1975), 359-368.

6. B. V. Gnedenko and A. N. Kolmogorov, Limit distributions for sums of independent random variables, Addison-Wesley, Reading, Mass., 1954.

7. E. Hille, Analytic function theory, Vol. 2, Ginn, Waltham, Mass., 1962.

8. N. Ikeda, M. Nagasawa and S. Watanabe, Branching Markov processes, I, II, III, J. Math. Kyoto Univ. 8 (1968), 233-278, 365-410, 9 (1969), 95-160.

9. S. Karlin, A first course in stochastic processes, Academic Press, New York, 1969.

10. E. Lukacs, Characteristic functions, Griffin, London, 1960.

11. J. E. Moyal, Discontinuous Markov processes, Acta Math. 98 (1967), 221-264.

12. T. W. Mullikan, Limiting distributions for critical multi-type branching processes with discrete time, Trans. Amer. Math. Soc. 106 (1963), 469-494.

13. Y. Ogura, Asymptotic behavior of multi-type Galton-Watson processes, J. Math. Kyoto Univ. 15 (1975), 251-302.

14. A. V. Skorokhod, Branching diffusion process, Theor. Probability Appl. 9 (1964), 492-497.

15. A. A. Savin and V. P. Chistyakov, Some theorems for branching processes with several types of particles, Theor. Probability Appl. 7 (1962), 93-100. 
16. S. Sawyer, A formula for semi-groups with an application to branching diffusion processes, Trans. Amer. Math. Soc. 152 (1970), 1-38.

17. B Branching diffusion processes in population genetics, Advances in Appl. Probability 8 (1976), 659-689.

18. S. Watanabe, On the branching processes for Brownian particles with an absorbing boundary, J. Math. Kyoto Univ. 4 (1965), 385-398.

Department of Mathematics, Wayne State University, Detrott, Michigan 48202 